\title{
Dual finite frames for vector spaces over an arbitrary field with applications
}

\author{
P. M. Morillas
}

\begin{abstract}
In the present paper, we study frames for finitedimensional vector spaces over an arbitrary field. We develop a theory of dual frames in order to obtain and study the different representations of the elements of the vector space provided by a frame. We relate the introduced theory with the classical one of dual frames for Hilbert spaces and apply it to study dual frames for three types of vector spaces: for vector spaces over conjugate closed subfields of the complex numbers (in particular, for cyclotomic fields), for metric vector spaces, and for ultrametric normed vector spaces over complete non-archimedean valued fields. Finally, we consider the matrix representation of operators using dual frames and its application to the solution of operators equations in a Petrov-Galerkin scheme.
\end{abstract}

Key Words: Vector Spaces, Fields, Dual Frames, Hilbert Spaces, Metric Vector Spaces, Ultrametric Normed Vector Spaces

Mathematics Subject Classification 2010: 42C15, 15A03, 15A63, 41A45

\section{Introduction}

Frames were introduced by Duffin and Schaeffer in 1952 for some Hilbert function spaces [20]. Their importance in data processing was established by Daubechies, Grossman and Meyer in [18] (see also [19]). Since then, the theory and applications of frames have been developed considerably. Today, the theory of frames in Hilbert spaces is well established. Some extensions to other vector spaces, such as Banach spaces, are also considered. Frames have various advantages over bases, which have made them very useful in several areas of sciences and engineering. Applications of frames include, e.g., signal and image processing, communication theory, coding theory, information theory and sampling theory, among others. For more details we refer the reader to [9, 11, 14, 28, 31] and references therein. 
The applications principally require a finite-dimensional setting. Therefore, to avoid the approximation problems related to the truncation needed in the infinite-dimensional case, it is important to work directly with finite frames. In this case, a frame is simply a spanning set. With a frame, each vector in the space has infinitely many representations. This redundancy is the key to their advantages over bases in applications. For example, when the independence requirement is removed, we have more flexibility to construct a frame suitable for the particular problem at hand. Moreover, frames provided resilience to, e.g., losses, additive noises and quantization errors.

The classical theory of finite frames had developed in finite-dimensional vector spaces $\mathcal{V}$ over the fields $\mathbb{R}$ and $\mathbb{C}$ (see [11, 28, 31, 35]). The vector spaces, mainly considered in the literature, are $\mathbb{R}^{d}$ and $\mathbb{C}^{d}$. Other considered vector spaces are: the complex vector space $\mathbb{C}^{\mathbb{Z}}$ of complex finite sequences over the field $\mathbb{Z}_{p}$ of the integers modulo $p\left[22\right.$, the complex vector space $\mathbb{C}^{G}$ of complex finite sequences over a finite cyclic group $G[24$ and the complex vector space $\mathbb{C}^{\mathbb{F}}$ of complex finite sequences over a finite field $\mathbb{F}[23]$.

In this paper, we consider frames for finite-dimensional vector spaces $\mathcal{V}$ over an arbitrary field $\mathbb{F}$. In order to study the representations of the elements in $\mathcal{V}$ provided by a frame, we introduce a concept of dual frame and analyze its properties. These dual frames are families of elements in a dual vector space $\mathcal{S}^{\prime}$, where $\mathcal{S}$ is a vector space that contains $\mathcal{V}$. If $\mathcal{F}$ is a basis, there is a unique dual of $\mathcal{F}$, which coincide with the classical dual basis.

The definition of dual frame introduced in the present paper can be viewed as an extension of one the presented in [36] (see also [13, 35]), where $\mathcal{S}=\mathcal{V}$ and only such fields $\mathbb{F}$ that $\mathbb{F} \subseteq \mathbb{C}$ and $\overline{\mathbb{F}}=\mathbb{F}$ are considered. With these restrictions on $\mathbb{F}$, an inner product on $\mathcal{V}$ and a canonical dual can be defined. This canonical dual is the object of study in these works. In our case, the field $\mathbb{F}$ is arbitrary, we do not deal with any inner product in $\mathcal{V}$, and we consider all possible duals of $\mathcal{F}$. Moreover, in our case $\mathcal{S} \supseteq \mathcal{V}$, and it results that oblique dual frames [15, 21] in Hilbert spaces are in the setting of our dual frames.

Working in a vector space over an arbitrary field provides a context of great generality. On the one hand, the theory of dual frames developed here gives the possibility to apply the representations provided by frames even in those areas where there is no other structure defined on the vector space. On the other hand, it allows considering dual frames for different vector spaces with additional structures. Here, we apply this theory to study dual frames for three particular vector spaces. We first look at the vector spaces considered in [13, 36, 35]. In particular, we analyze the existence of dual frames for cyclotomic fields with some of the properties of the canonical dual. These properties are useful for computational purposes. Then we analyze dual frames for metric vector spaces, which are a natural generalization of 
real inner product spaces. We apply our results to have a theory of dual frames for these vector spaces and compare it with the classical theory in Hilbert spaces. Note that frames and Parseval frames for the metric vector space $\mathbb{Z}_{2}^{d}$ were considered first in [7] and studied later in, e.g., [8, 27, 29]. Other related articles are [26, 25], where frames over finite fields in unitary and orthogonal geometries are considered. Finally, we study frames and dual frames for ultrametric normed vector spaces focusing principally on perturbations results. As another application of the developed theory, the frame representation of operators and the solution of operator equations in a Petrov-Galerkin scheme are considered. These operator equations can be of different types, such as differential equations or integral equations.

The organization of the paper is as follows. In Section 1, we introduce some notations. In section 2, we discuss the concept of frame for finitedimensional vector spaces over an arbitrary field. We also consider frames for dual spaces. Some fundamental properties are established. In section 3, we introduce and study the concept of dual frame. We give several characterizations. Section 4 is devoted to the construction of all dual frames of a given frame, whereas Section 5 deals with the construction of specific dual frames. In these sections, the general theory is related to the classical theory of dual frames for Hilbert spaces. In Section 6, the obtained results are used to investigate the dual frames of perturbed frames in the classical setting. Different types of perturbations are considered: close frames, near frames and Paley-Wiener perturbations. In Section 7, we apply the developed theory to analyze frames and dual frames for three particular vector spaces: vector spaces over $\mathbb{F}$ where $\mathbb{F} \subseteq \mathbb{C}$ and $\overline{\mathbb{F}}=\mathbb{F}$, metric vector spaces and ultrametric normed vector spaces over complete non-archimedean valued fields. We also consider the representation of operators using dual frames and their application to the solution of operator equations.

\section{Preliminares}

In this paper, we will use well-known notions from advanced linear algebra. We refer the reader to, e.g., 33 for more details.

Let $\mathbb{F}$ be an arbitrary field. Let $\mathcal{R}$ and $\mathcal{S}$ be vector spaces over $\mathbb{F}, \mathcal{W}$ be a finite-dimensional subspace of $\mathcal{R}$ and $\mathcal{V}$ be a finite-dimensional subspace of $\mathcal{S}$.

We denote the space of linear transformations from $\mathcal{R}$ to $\mathcal{S}$ by $L(\mathcal{R}, \mathcal{S})$. We write $L(\mathcal{S})$ for $L(\mathcal{S}, \mathcal{S})$. Given $T \in L(\mathcal{R}, \mathcal{S})$, we write $\operatorname{im}(T)$ and $\operatorname{ker}(T)$ to denote the image and the null space of $T$, respectively.

Given any (algebraic) complement $\mathcal{V}^{c}$ of $\mathcal{V}$ in $\mathcal{S}$, we denote the (oblique) projection onto $\mathcal{V}$ along $\mathcal{V}^{c}$ with $P \mathcal{V}, \mathcal{V}^{c}$. Recall that a linear operator $T \in L(\mathcal{S})$ 
is idempotent, i.e., $T^{2}=T$, if and only if

$$
\mathcal{S}=\operatorname{im}(T) \oplus \operatorname{ker}(T) \quad \text { and } \quad T=P_{\operatorname{im}(T), \operatorname{ker}(T)}
$$

Let $T \in L(\mathcal{R}, \mathcal{S})$ with $\operatorname{im}(T)=\mathcal{V}$ and $\operatorname{ker}(T)=\mathcal{W}$. Let $\mathcal{W}^{c}$ be any complement of $\mathcal{W}$ in $\mathcal{R}$ and $\mathcal{V}^{c}$ be any complement of $\mathcal{V}$ in $\mathcal{S}$. We denote the oblique pseudoinverse of $T$ on $\mathcal{W}^{c}$ along $\mathcal{V}^{c}$ by $T_{\mathcal{W}^{c}, \mathcal{V}^{c}}^{\sharp}$. Recall that $T_{\mathcal{W}^{c}, \mathcal{V}^{c}}^{\sharp} \in L(\mathcal{S}, \mathcal{R})$ is given by

$$
\left(T_{\mathcal{W}^{c}, \mathcal{V}^{c}}^{\sharp}\right)_{\mid \mathcal{V}}=\left(T_{\mid \mathcal{W}^{c}}\right)^{-1} \quad \text { and } \quad \operatorname{ker}\left(T_{\mathcal{W}^{c}, \mathcal{V}^{c}}^{\sharp}\right)=\mathcal{V}^{c} .
$$

The oblique pseudoinverse $T_{\mathcal{W}^{c}, \mathcal{V}^{c}}^{\sharp}$ is characterized by the following conditions:

$$
T_{\mathcal{W}^{c}, \mathcal{V}^{c}}^{\sharp} T=P_{\mathcal{W}^{c}, \mathcal{W}}, \quad T T_{\mathcal{W}^{c}, \mathcal{V}^{c}}^{\sharp}=P_{\mathcal{V}, \mathcal{V}^{c}} \quad \text { and } \quad \operatorname{im}\left(T_{\mathcal{W}^{c}, \mathcal{V}^{c}}^{\sharp}\right)=\mathcal{W}^{c} .
$$

The (algebraic) dual space of $\mathcal{S}$ will be denoted by $\mathcal{S}^{\prime}$. For $f \in \mathcal{V}, \widehat{f} \in \mathcal{V}^{\prime \prime}$ is given by $\widehat{f}(\phi)=\phi(f)$ for each $\phi \in \mathcal{V}^{\prime}$. Recall that $\mathcal{V} \approx \mathcal{V}^{\prime \prime}$.

Let $I$ and $J$ be any finite sets. The standard basis of $\mathbb{F}^{I}$ will be denoteb by $\left\{\delta_{i}\right\}_{i \in I}$. If $x \in \mathbb{F}^{I}, x(k)$ denotes its $k$-th component. For each $i \in I$, we denote the $i$-th canonical functional on $\mathbb{F}^{I}$ by $p_{i}$, i.e., $p_{i} x=x(i)$ for each $x \in \mathbb{F}^{I}$.

\section{Frames for finite-dimensional vector spaces}

In what follows, we assume that $\mathcal{S}$ is a vector space over $\mathbb{F}$ and that $\mathcal{V}$ is a subspace of $\mathcal{S}$ of finite dimension. Let $\mathcal{F}=\left\{f_{i}\right\}_{i \in I} \subseteq \mathcal{V}$. The synthesis operator of $\mathcal{F}$ is

$$
T_{\mathcal{F}}: \mathbb{F}^{I} \rightarrow \mathcal{V}, \quad T_{\mathcal{F}} x=\sum_{i \in I} x(i) f_{i} .
$$

Recall the definition of frame in a finite-dimensional vector space:

Definition 1 Let $\mathcal{F}=\left\{f_{i}\right\}_{i \in I} \subseteq \mathcal{V}$. We say that $\mathcal{F}$ is a frame for $\mathcal{V}$ if $\operatorname{span} \mathcal{F}=\mathcal{V}$.

Observe that $\mathcal{F} \subseteq \mathcal{V}$ is a frame for $\mathcal{V}$ if and only if $\operatorname{im}\left(T_{\mathcal{F}}\right)=\mathcal{V}$. Frames have some advantages over bases. Since we are not considering, as for bases, the linear independence condition, we have more freedom to design frames to fit the requirements of an application at hand. Moreover, a basis ceases to be a basis if one element is added or removed, but a frame remains a frame if some elements are added and can still be a frame if some elements are 
removed. Another advantage is the following. Assume that $f=\sum_{i \in I} c(i) f_{i}$ and that the coefficients are perturbed by $w \in \mathbb{F}^{I}$ in such a way that we get

$$
\sum_{i \in I}(c(i)+w(i)) f_{i}=f+\sum_{i \in I} w(i) f_{i}
$$

If the perturbation contribution $\sum_{i \in I} w(i) f_{i}$ is zero we can even recover $f$. Now, if $\left\{f_{i}\right\}_{i \in I}$ is overcomplete, we can obtain $f=\sum_{i \in I}(c(i)+w(i)) f_{i}$ if $w$ falls into the nontrivial kernel of the synthesis operator $T_{\mathcal{F}}$. If $\left\{f_{i}\right\}_{i \in I}$ is a basis, this never happen.

Now we consider frames for dual spaces. Let $\Phi=\left\{\phi_{j}\right\}_{j \in J} \subseteq \mathcal{S}^{\prime}$. The analysis operator of $\Phi$ is

$$
T_{\Phi}^{*}: \mathcal{S} \rightarrow \mathbb{F}^{J}, \quad T_{\Phi}^{*} f=\left(\phi_{j}(f)\right)_{j \in J} .
$$

We denote $\left\{\left(\phi_{j}\right)_{\mid \mathcal{V}}\right\}_{j \in J}$ by $\Phi_{\mid \mathcal{V}}$. The next theorem characterizes frames for dual spaces.

Theorem 1 Let $\Phi=\left\{\phi_{j}\right\}_{j \in J} \subseteq \mathcal{S}^{\prime}$. Then $\Phi_{\mid \mathcal{V}}$ is a frame for $\mathcal{V}^{\prime}$ if and only if $\mathcal{V} \cap \operatorname{ker}\left(T_{\Phi}^{*}\right)=\{0\}$ or, equivalently, $T_{\Phi}^{*}{ }_{\mid \mathcal{V}}$ is injective.

Proof. Suppose that $\Phi_{\mid \mathcal{V}}$ is a frame for $\mathcal{V}^{\prime}$ and $f \in \mathcal{V}$ is such that $T_{\Phi}^{*}(f)=0$, i.e.,

$$
\phi_{j}(f)=0 \quad \text { for each } j \in J .
$$

Since $\left\{\left(\phi_{j}\right)_{\mid \mathcal{V}}\right\}_{j \in J}$ spans $\mathcal{V}^{\prime}$,

$$
\lambda(f)=0 \quad \text { for each } \lambda \in \mathcal{V}^{\prime} .
$$

Then $f=0$. This shows that $\mathcal{V} \cap \operatorname{ker}\left(T_{\Phi}^{*}\right)=\{0\}$.

Conversely, assume that $\mathcal{V} \cap \operatorname{ker}\left(T_{\Phi}^{*}\right)=\{0\}$ or, equivalently, $T_{\Phi}^{*}$ is injective. Let $A$ be a linear left inverse of $T_{\Phi_{\mid \mathcal{V}}}^{*}$. If $f \in \mathcal{V}$, we have

$$
f=A T_{\Phi}^{*}(f)=\sum_{j \in J} \phi_{j}(f) A\left(\delta_{j}\right)
$$

Therefore, if $\lambda \in \mathcal{V}^{\prime}$, then

$$
\lambda=\sum_{j \in J} \lambda\left(A\left(\delta_{j}\right)\right)\left(\phi_{j}\right)_{\mid \mathcal{V}}
$$

This proves that $\left\{\left(\phi_{j}\right)_{\mid \mathcal{V}}\right\}_{j \in J}$ spans $\mathcal{V}^{\prime}$, i.e., $\Phi_{\mid \mathcal{V}}$ is a frame for $\mathcal{V}^{\prime}$

Example 1 Let $\mathcal{S}$ be a finite-dimensional real or complex inner product vector space. Let $\mathcal{F}=\left\{f_{i}\right\}_{i \in I} \subset \mathcal{S}$. Then $\mathcal{F}$ is a frame for $\mathcal{V}$ if and only if the frame operator

$$
S_{\mathcal{F}}:=T_{\mathcal{F}} T_{\mathcal{F}}^{*}
$$


is invertible. Here $T_{\mathcal{F}}^{*}: \mathcal{S} \rightarrow \mathbb{F}^{I}$ given by

$$
T_{\mathcal{F}}^{*} f=\left(\left\langle f, f_{i}\right\rangle\right)_{i \in I} \quad \text { for each } f \in \mathcal{S},
$$

is the adjoint of $T_{\mathcal{F}}$, where $T_{\mathcal{F}}$ is considered as an element of $L\left(\mathbb{F}^{I}, \mathcal{S}\right)$.

If $\mathcal{S}$ is a finite-dimensional real or complex inner product vector space, then each $\phi \in \mathcal{S}^{\prime}$ has a Riesz representation $\phi(f)=\langle f, g\rangle$ for some $g \in \mathcal{S}$. Let $\Phi=\left\{\phi_{j}\right\}_{j \in J} \subset \mathcal{S}^{\prime}$ and let $g_{j} \in \mathcal{S}$ correspond to $\phi_{j} \in \mathcal{S}^{\prime}$ for each $j \in J$. Let

$$
\mathcal{W}:=\operatorname{span}\left\{g_{j}\right\}_{j \in J}
$$

By Theorem 1, $\Phi_{\mid \mathcal{V}}$ is a frame for $\mathcal{V}^{\prime}$ if and only if $\mathcal{V} \cap \mathcal{W}^{\perp}=\{0\}$.

For $\mathcal{F}=\left\{f_{i}\right\}_{i \in I} \subseteq \mathcal{V}$, we define the linear transformations

$$
T_{\widehat{\mathcal{F}}}: \mathbb{F}^{I} \rightarrow \mathcal{V}^{\prime \prime}, \quad T_{\widehat{\mathcal{F}}} x=\sum_{i \in I} x(i) \widehat{f}_{i}
$$

and

$$
T_{\widehat{\mathcal{F}}}^{*}: \mathcal{V}^{\prime} \rightarrow \mathbb{F}^{I}, \quad T_{\widehat{\mathcal{F}}}^{*} \lambda=\left(\widehat{f}_{i}(\lambda)\right)_{i \in I}=\left(\lambda\left(f_{i}\right)\right)_{i \in I} .
$$

Proposition 1 Let $\mathcal{F}=\left\{f_{i}\right\}_{i \in I} \subseteq \mathcal{V}$. The following assertions are equivalent:

(i) $\mathcal{F}$ is a frame for $\mathcal{V}$.

(ii) $\widehat{\mathcal{F}}$ is a frame for $\mathcal{V}^{\prime \prime}$.

(iii) $T_{\widehat{\mathcal{F}}}$ is onto.

(iv) $T_{\widehat{\mathcal{F}}}^{*}$ is injective.

Proof. (i) $\Rightarrow$ (ii). Suppose that $\mathcal{F}$ is a frame for $\mathcal{V}$. Let $\lambda \in \mathcal{V}^{\prime \prime}$ and $f \in \mathcal{V}$ be such that $\lambda=\widehat{f}$. Since $\mathcal{F}$ is a frame for $\mathcal{V}$, there exists $x \in \mathbb{F}^{I}$ such that $f=\sum_{i \in I} x(i) f_{i}$. For each $\phi \in \mathcal{V}^{\prime}$, we have

$$
\lambda(\phi)=\widehat{f}(\phi)=\phi(f)=\sum_{i \in I} x(i) \phi\left(f_{i}\right)=\sum_{i \in I} x(i) \widehat{f}_{i}(\phi) .
$$

This shows that $\widehat{\mathcal{F}}$ is a frame for $\mathcal{V}^{\prime \prime}$.

(ii) $\Rightarrow$ (i). Suppose that $\widehat{\mathcal{F}}$ is a frame for $\mathcal{V}^{\prime \prime}$. Let $f \in \mathcal{V}$. Then there exists $x \in \mathbb{F}^{I}$ such that $\widehat{f}=\sum_{i \in I} x(i) \widehat{f}_{i}$. Thus,

$$
\lambda(f)=\sum_{i \in I} x(i) \lambda\left(f_{i}\right) \quad \text { for all } \lambda \in \mathcal{V}^{\prime} .
$$

Then $f=\sum_{i \in I} x(i) f_{i}$. From this, $\mathcal{F}$ is a frame for $\mathcal{V}$.

(ii) $\Leftrightarrow$ (iii). It is immediate.

(ii) $\Leftrightarrow$ (iv). It is similar to the proof of Theorem 1 . 
For future references, we enunciate the following basic lemma.

Lemma 1 Let $\mathcal{F}=\left\{f_{i}\right\}_{i \in I} \subseteq \mathcal{V}$ and $\Phi=\left\{\phi_{j}\right\}_{j \in J} \subseteq \mathcal{S}^{\prime}$. The following assertions hold:

(i) If $\operatorname{im}\left(T_{\mathcal{F}}\right)=\mathcal{V}$, then $\operatorname{im}\left(T_{\Phi}^{*} T_{\mathcal{F}}\right)=\operatorname{im}\left(T_{\Phi_{\mid \mathcal{V}}}^{*}\right)$.

(ii) If $T_{\Phi_{\mid \mathcal{V}}}^{*}$ is injective, then $\operatorname{ker}\left(T_{\Phi}^{*} T_{\mathcal{F}}\right)=\operatorname{ker}\left(T_{\mathcal{F}}\right)$.

\subsection{Linear dependencies}

Given $\mathcal{F}=\left\{f_{i}\right\}_{i \in I} \subseteq \mathcal{V}$ we call $\operatorname{ker}\left(T_{\mathcal{F}}\right)$ the set of linear dependencies of $\mathcal{F}$ and we denote it with $\operatorname{dep}(\mathcal{F})$. This nomenclature is used in the particular context of [36].

Proposition 2 Let $\mathcal{F}=\left\{f_{i}\right\}_{i \in I} \subseteq \mathcal{V}$. Then $\operatorname{dep}(\mathcal{F})=\operatorname{dep}(\widehat{\mathcal{F}})$.

Proof. Let $c \in \mathbb{F}^{I}$. Then

$$
T_{\mathcal{F}} c=\sum_{i \in I} c(i) f_{i}=0
$$

if and only if

$$
\sum_{i \in I} c(i) \widehat{f}_{i}(\lambda)=\sum_{i \in I} c(i) \lambda\left(f_{i}\right)=0 \quad \text { for all } \lambda \in \mathcal{V}^{\prime}
$$

This last condition is equivalent to

$$
T_{\widehat{\mathcal{F}}} c=\sum_{i \in I} c(i) \widehat{f}_{i}=0
$$

Hence, $\operatorname{dep}(\mathcal{F})=\operatorname{dep}(\widehat{\mathcal{F}})$.

Now we consider the concept of similarity for frames.

Definition 2 Let $\mathcal{F}=\left\{f_{i}\right\}_{i \in I} \subseteq \mathcal{V}, \mathcal{G}=\left\{g_{i}\right\}_{i \in I} \subseteq \mathcal{W}$ and $\operatorname{dim}(\mathcal{V})=$ $\operatorname{dim}(\mathcal{W})$. We say that $\mathcal{F}$ and $\mathcal{G}$ are similar if there exists an invertible transformation $Q \in L(\mathcal{V}, \mathcal{W})$ such that $Q f_{i}=g_{i}$ for each $i \in I$.

Example 2 Let $\mathcal{S}$ be a finite-dimensional real or complex inner product vector space. Let $\mathcal{F}=\left\{f_{i}\right\}_{i \in I}$ and $\mathcal{G}=\left\{g_{i}\right\}_{i \in I}$ be frames for $\mathcal{V}$. Then $\mathcal{F}$ and $\mathcal{G}$ are similar if and only if $T_{\mathcal{F}} T_{\mathcal{G}}^{*}$ is invertible. See [35, Proposition 3.2] for other conditions equivalent to similarity in this context.

The following lemma relates similarity with linear dependencies. 
Lemma 2 Let $\mathcal{F}=\left\{f_{i}\right\}_{i \in I}$ be a frame for $\mathcal{V}, \mathcal{G}=\left\{g_{i}\right\}_{i \in I}$ be a frame for $\mathcal{W}$ and $\operatorname{dim}(\mathcal{V})=\operatorname{dim}(\mathcal{W})$. Then the following are equivalent:

(i) $\mathcal{F}$ and $\mathcal{G}$ are similar.

(ii) $\operatorname{dep}(\mathcal{F})=\operatorname{dep}(\mathcal{G})$.

(iii) There is a common complement $\mathcal{C}$ of $\operatorname{dep}(\mathcal{F})$ and $\operatorname{dep}(\mathcal{G})$ in $\mathbb{F}^{I}$ such that $P_{\mathcal{C}, \operatorname{dep}(\mathcal{F})}=P_{\mathcal{C}, \operatorname{dep}(\mathcal{G})}$.

Proof. (i) $\Rightarrow$ (ii). Suppose that $\mathcal{F}$ and $\mathcal{G}$ are similar, i.e., there exists an invertible linear transformation $Q: \mathcal{V} \rightarrow \mathcal{W}$ such that

$$
Q f_{i}=g_{i} \quad \text { for each } i \in I \text {. }
$$

Then $T_{\mathcal{G}}=Q T_{\mathcal{F}}$ and $\operatorname{dep}(\mathcal{G})=\operatorname{dep}(\mathcal{F})$.

(ii) $\Rightarrow$ (i). Suppose now that $\operatorname{dep}(\mathcal{F})=\operatorname{dep}(\mathcal{G})$. Let $\mathcal{C}$ be a complement of $\operatorname{dep}(\mathcal{F})=\operatorname{dep}(\mathcal{G})$ in $\mathbb{F}^{I}$. Since $\left.T_{\mathcal{F}}\right|_{\mathcal{C}}: \mathcal{C} \rightarrow \mathcal{V}$ and $\left.T_{\mathcal{G}}\right|_{\mathcal{C}}=\left.T_{\mathcal{G}}\right|_{\mathcal{C}}: \mathcal{C} \rightarrow \mathcal{W}$ are bijections,

$$
Q=T_{\mathcal{G}}\left(\left.T_{\mathcal{F}}\right|_{\mathcal{C}}\right)^{-1}: \mathcal{V} \rightarrow \mathcal{W}
$$

is an invertible linear transformation. For each $i \in I$ we have

$$
Q f_{i}=T_{\mathcal{G}}\left(\left.T_{\mathcal{F}}\right|_{\mathcal{C}}\right)^{-1} f_{i}=T_{\mathcal{G}}\left(\left.T_{\mathcal{F}}\right|_{\mathcal{C}}\right)^{-1} T_{\mathcal{F}} P_{\mathcal{C}, \operatorname{dep}(\mathcal{F})} \delta_{i}=T_{\mathcal{G}} P_{\mathcal{C}, \operatorname{dep}(\mathcal{G})} \delta_{i}=T_{\mathcal{G}} \delta_{i}=g_{i}
$$

This shows that $\mathcal{F}$ and $\mathcal{G}$ are similar.

(ii) $\Leftrightarrow$ (iii). It is immediate.

Remark 1 (i) Let $\mathcal{F}=\left\{f_{i}\right\}_{i \in I}$ be a frame for $\mathcal{V}$. Let $\mathcal{R}$ be a vector space over $\mathbb{F}$ and $L \in L(\mathcal{V}, \mathcal{R})$. Then $\operatorname{dep}(\mathcal{F})=\operatorname{dep}(L \mathcal{F})$ if and only if $L$ is injective.

(ii) Let $\mathcal{F}=\left\{f_{i}\right\}_{i \in I} \subseteq \mathcal{V}$ and $\mathcal{G}=\left\{g_{i}\right\}_{i \in I} \subseteq \mathcal{V}$. Let $\mathcal{A}$ and $\mathcal{B}$ be complements of $\operatorname{dep}(\mathcal{F})$ and $\operatorname{dep}(\mathcal{G})$ in $\mathbb{F}^{I}$, respectively. Then $\operatorname{dep}(\mathcal{F}) \subseteq$ $\operatorname{dep}(\mathcal{G})$ if and only if $P_{\mathcal{B}, \operatorname{dep}(\mathcal{G})} P_{\operatorname{dep}(\mathcal{F}), \mathcal{A}}=0$. This last equality is equivalent to $P_{\mathcal{B}, \operatorname{dep}(\mathcal{G})} P_{\mathcal{A}, \operatorname{dep}(\mathcal{F})}=P_{\mathcal{B}, \operatorname{dep}(\mathcal{G})}$.

By a proof similar to that of Lemma 2, we can see that $\operatorname{dep}(\mathcal{F}) \subseteq \operatorname{dep}(\mathcal{G})$ if and only if there exists a surjective transformation $Q \in L(\mathcal{V}, \mathcal{W})$ such that $Q f_{i}=g_{i}$, for each $i \in I$. In this case, for the "only if" part we consider $Q=T_{\mathcal{G}}\left(\left.T_{\mathcal{F}}\right|_{\operatorname{dep}(\mathcal{F})^{c}}\right)^{-1}$ where $\operatorname{dep}(\mathcal{F})^{c}$ is any complement of $\operatorname{dep}(\mathcal{F})$.

(iii) If $\mathbb{F} \subseteq \mathbb{C}, \overline{\mathbb{F}}=\mathbb{F}$ and $\mathcal{C}=\operatorname{dep}(\mathcal{F})^{\perp}$, then Lemma 2 reduced to [36, Lemma 3.3].

Remark 2 Let $\operatorname{dep}(\mathcal{F})^{c}$ be a subspace of $\mathbb{F}^{I}$ such that $\mathbb{F}^{I}=\operatorname{dep}(\mathcal{F}) \oplus$ $\operatorname{dep}(\mathcal{F})^{c}$. Then

$$
\left\{P_{\operatorname{dep}(\mathcal{F})^{c}, \operatorname{dep}(\mathcal{F})} \delta_{i}\right\}_{i \in I}
$$


is a frame for $\operatorname{dep}(\mathcal{F})^{c}$ with synthesis operator $P_{\operatorname{dep}(\mathcal{F})^{c}, \operatorname{dep}(\mathcal{F}) \text {. As a conse- }}$ quence of Lemma 2, this frame is similar to $\mathcal{F}$. If $\mathcal{F}$ is a basis for $\mathcal{V}$, then $\operatorname{dep}(\mathcal{F})=\{0\}, \operatorname{dep}(\mathcal{F})^{c}=\mathbb{F}^{I}$ and $P_{\operatorname{dep}(\mathcal{F})^{c}, \operatorname{dep}(\mathcal{F})} \delta_{i}=\delta_{i}$ for each $i \in I$.

If we consider any two complements $\mathcal{C}_{1}$ and $\mathcal{C}_{2}$ of $\operatorname{dep}(\mathcal{F})$ in $\mathbb{F}^{I}$, then the frames $\left\{P_{\mathcal{C}_{1}, \operatorname{dep}(\mathcal{F})} \delta_{i}\right\}_{i \in I}$ and $\left\{P_{\mathcal{C}_{2}, \operatorname{dep}(\mathcal{F})} \delta_{i}\right\}_{i \in I}$ are similar and we can take any of them as a representative in the class of frames similar to $\mathcal{F}$.

Example 3 Let $\mathcal{S}$ be a finite-dimensional real or complex inner product vector space. Let $\mathcal{F}=\left\{f_{i}\right\}_{i \in I}$ be a frame for $\mathcal{V}$. Then $P_{\operatorname{dep}(\mathcal{F})^{\perp}, \operatorname{dep}(\mathcal{F})}=$ $T_{\mathcal{F}}^{*} S_{\mathcal{F}}^{-1} T_{\mathcal{F}}$ [35, Example 4.3] and $\left\{T_{\mathcal{F}}^{*} S_{\mathcal{F}}^{-1} T_{\mathcal{F}} \delta_{i}\right\}_{i \in I}=\left\{\left(\left\langle S_{\mathcal{F}}^{-1} f_{i}, f_{j}\right\rangle\right)_{j \in I}\right\}_{i \in I}=$ $\left\{\left(\left\langle S_{\mathcal{F}}^{-1 / 2} f_{i}, S_{\mathcal{F}}^{-1 / 2} f_{j}\right\rangle\right)_{j \in I}\right\}_{i \in I}$ is the canonical representative in the class of frames similar to $\mathcal{F}$.

\section{Dual frames}

In this section, we introduce the concept of dual frame which allows us to obtain representations of the elements of a vector space given by a frame. This concept enables the study and the application of these representations.

Definition 3 Let $\mathcal{F}=\left\{f_{i}\right\}_{i \in I} \subseteq \mathcal{V}$ and $\Phi=\left\{\phi_{i}\right\}_{i \in I} \subseteq \mathcal{S}^{\prime}$. We say that $\mathcal{F}$ and $\Phi$ are dual in $\mathcal{V}$ if $f=T_{\mathcal{F}} T_{\Phi}^{*} f$ for all $f \in \mathcal{V}$.

In the above definition, the family $\Phi$ gives the coefficients needed to represent each element in $\mathcal{V}$ using the frame $\mathcal{F}$ (see also Theorem 2(ii) below). In what follows, we analyze different properties of these families in order to study and apply the representations provided by a frame.

Example 4 Let $\mathcal{S}$ be a finite-dimensional real or complex inner product vector space. Using the identification of $\mathcal{S}^{\prime}$ with $\mathcal{S}$, Definition 3 becomes: Let $\mathcal{F}=\left\{f_{i}\right\}_{i \in I} \subset \mathcal{V}$ and $\mathcal{G}=\left\{g_{i}\right\}_{i \in I} \subset \mathcal{S}$. We say that $\mathcal{F}$ and $\mathcal{G}$ are dual in $\mathcal{V}$ if

$$
f=T_{\mathcal{F}} T_{\mathcal{G}}^{*} f=\sum_{i \in I}\left\langle f, g_{i}\right\rangle f_{i} \quad \text { for each } f \in \mathcal{V} .
$$

Here $T_{\mathcal{G}}^{*}: \mathcal{S} \rightarrow \mathbb{F}^{I}$ given by

$$
T_{\mathcal{G}}^{*} f=\left(\left\langle f, g_{i}\right\rangle\right)_{i \in I} \quad \text { for each } f \in \mathcal{S},
$$

is the adjoint of $T_{\mathcal{F}}$, where $T_{\mathcal{F}}$ is considered as an element of $L\left(\mathbb{F}^{I}, \mathcal{S}\right)$.

The following theorem characterizes dual frames.

Theorem 2 Let $\mathcal{F}=\left\{f_{i}\right\}_{i \in I} \subseteq \mathcal{V}$ and $\Phi=\left\{\phi_{i}\right\}_{i \in I} \subseteq \mathcal{S}^{\prime}$. Then the following statements are equivalent: 
(i) $\mathcal{F}$ and $\Phi$ are dual in $\mathcal{V}$.

(ii) $f=\sum_{i \in I} \phi_{i}(f) f_{i}$ for all $f \in \mathcal{V}$.

(iii) $\Phi$ and $\widehat{\mathcal{F}}$ are dual in $\mathcal{V}^{\prime}$, i.e., $\lambda=T_{\Phi} T_{\widehat{\mathcal{F}}}^{*} \lambda$ for all $\lambda \in \mathcal{V}^{\prime}$.

(iv) $\lambda=\sum_{i \in I} \lambda\left(f_{i}\right) \phi_{i}$ for all $\lambda \in \mathcal{V}^{\prime}$.

(v) $\widehat{f}(\lambda)=\sum_{i \in I} \lambda\left(f_{i}\right) \widehat{f}\left(\phi_{i}\right)$ for all $f \in \mathcal{V}$ and all $\lambda \in \mathcal{V}^{\prime}$.

(vi) $i m\left(T_{\mathcal{F}}\right)=\mathcal{V}, T_{\Phi}^{*}$ is injective and $\left(T_{\Phi}^{*} T_{\mathcal{F}}\right)^{2}=T_{\Phi}^{*} T_{\mathcal{F}}$.

(vii) $i m\left(T_{\mathcal{F}}\right)=\mathcal{V}$ and $T_{\mathcal{F}}\left(T_{\Phi}^{*} T_{\mathcal{F}}\right)=T_{\mathcal{F}}$.

(viii) $T_{\Phi_{\mid \mathcal{V}}}^{*}$ is injective and $\left(T_{\Phi}^{*} T_{\mathcal{F}}\right) T_{\Phi_{\mid \mathcal{V}}}^{*}=T_{\Phi_{\mid \mathcal{V}}}^{*}$.

(ix) $i m\left(T_{\mathcal{F}}\right)=\mathcal{V}, \mathbb{F}^{I}=\operatorname{im}\left(T_{\Phi_{\mid \mathcal{V}}}^{*}\right) \oplus \operatorname{dep}(\mathcal{F})$ and $\left.T_{\Phi}^{*} T_{\mathcal{F}}=P_{\operatorname{im}\left(T_{\Phi}^{*}{ }_{\mathcal{V}}\right.}\right) \operatorname{dep}(\mathcal{F})$.

(x) $i m\left(T_{\mathcal{F}}\right)=\mathcal{V}, T_{\Phi_{\mid \mathcal{V}}}^{*}$ is injective and the matrix $\left(\phi_{j}\left(f_{i}\right)\right)_{j, i \in I}$ has eigenvector $\left(\phi_{j}\left(f_{i}\right)\right)_{j \in I}$ with eigenvalue 1 for all $i \in I$ such that $f_{i} \neq 0$.

Proof. The equivalence of (i)-(v) is immediate.

(i) $\Rightarrow$ (vi),(vii),(viii). Suppose that (i) holds. If $f \in \mathcal{V}$, then

$$
f=T_{\mathcal{F}} T_{\Phi}^{*} f .
$$

Thus, $\operatorname{im}\left(T_{\mathcal{F}}\right)=\mathcal{V}$. If $f \in \mathcal{V} \cap \operatorname{ker}\left(T_{\Phi}^{*}\right)$, then

$$
f=T_{\mathcal{F}} T_{\Phi}^{*} f=0 .
$$

Hence, $T_{\Phi_{\mid \mathcal{V}}}^{*}$ is injective. Now let $x \in \mathbb{F}^{I}$ and $f \in \mathcal{V}$. We have

$$
\begin{gathered}
\left(T_{\Phi}^{*} T_{\mathcal{F}}\right)^{2} x=T_{\Phi}^{*}\left(T_{\mathcal{F}} T_{\Phi}^{*}\right) T_{\mathcal{F}} x=T_{\Phi}^{*} T_{\mathcal{F}} x \\
T_{\mathcal{F}}\left(T_{\Phi}^{*} T_{\mathcal{F}}\right) x=\left(T_{\mathcal{F}} T_{\Phi}^{*}\right) T_{\mathcal{F}} x=T_{\mathcal{F}} x
\end{gathered}
$$

and

$$
\left(T_{\Phi}^{*} T_{\mathcal{F}}\right) T_{\Phi}^{*} f=T_{\Phi}^{*}\left(T_{\mathcal{F}} T_{\Phi}^{*}\right) f=T_{\Phi}^{*} f .
$$

Thus, (vi),(vii) and (viii) are satisfied.

(vi) $\Rightarrow$ (i). Suppose that (vi) holds. Let $f \in \mathcal{V}$. Since $\operatorname{im}\left(T_{\mathcal{F}}\right)=\mathcal{V}$, there exists $x \in \mathbb{F}^{I}$ such that $f=T_{\mathcal{F}} x$. Thus,

$$
T_{\mathcal{F}} T_{\Phi}^{*} f=T_{\mathcal{F}} T_{\Phi}^{*} T_{\mathcal{F}} x .
$$

From here,

$$
T_{\Phi}^{*} T_{\mathcal{F}} T_{\Phi}^{*} f=T_{\Phi}^{*} T_{\mathcal{F}} T_{\Phi}^{*} T_{\mathcal{F}} x=T_{\Phi}^{*} T_{\mathcal{F}} x=T_{\Phi}^{*} f .
$$

Since $T_{\Phi}^{*}$ is injective, $T_{\mathcal{F}} T_{\Phi}^{*} f=f$. This shows that (i) holds. 
(vii) $\Rightarrow$ (i). Let $f \in \mathcal{V}$. If (vii) holds, there exists $x \in \mathbb{F}^{I}$ such that

$$
f=T_{\mathcal{F}} x=T_{\mathcal{F}}\left(T_{\Phi}^{*} T_{\mathcal{F}}\right) x=\left(T_{\mathcal{F}} T_{\Phi}^{*}\right) T_{\mathcal{F}} x=T_{\mathcal{F}} T_{\Phi}^{*} f .
$$

Thus, (i) holds.

(viii) $\Rightarrow$ (i). Let $f \in \mathcal{V}$. If (viii) holds, then

$$
\left(T_{\Phi}^{*} T_{\mathcal{F}}\right) T_{\Phi}^{*} f=T_{\Phi}^{*} f
$$

and the injectivity of $T_{\Phi_{\mid \mathcal{V}}}^{*}$ assure that

$$
T_{\mathcal{F}} T_{\Phi}^{*} f=f
$$

and (i) holds.

(vi) $\Rightarrow$ (ix). If (vi) is satisfied, then $\operatorname{im}\left(T_{\mathcal{F}}\right)=\mathcal{V}$ and, by Lemma 1 ,

$$
\mathbb{F}^{I}=\operatorname{im}\left(T_{\Phi_{\mid \mathcal{V}}}^{*}\right) \oplus \operatorname{dep}(\mathcal{F}) \quad \text { and } \quad T_{\Phi}^{*} T_{\mathcal{F}}=P_{\operatorname{im}\left(\left.T_{\Phi}\right|_{\mathcal{V}}\right) \operatorname{dep}(\mathcal{F})} .
$$

This shows (ix).

(ix) $\Rightarrow$ (vii) is immediate.

(vi) $\Leftrightarrow(\mathrm{x})$. The matrices of $T_{\Phi}^{*} T_{\mathcal{F}}$ and $\left(T_{\Phi}^{*} T_{\mathcal{F}}\right)^{2}$ in the standard basis of $\mathbb{F}^{I}$ have entry $(j, i)$ equal to $\phi_{j}\left(f_{i}\right)$ and $\sum_{k \in I} \phi_{j}\left(f_{k}\right) \phi_{k}\left(f_{i}\right)$, respectively. From here, the equivalence of (vi) and (x) follows.

Remark 3 Definition 3 is a generalization of the one considered in [36] where $\mathcal{V}=\mathcal{S}, \mathbb{F} \subseteq \mathbb{C}, \overline{\mathbb{F}}=\mathbb{F}, \mathcal{F}=\left\{f_{i}\right\}_{i \in I}$ is a frame for $\mathcal{S}$ and $\Phi=\left\{\phi_{i}\right\}_{i \in I}$ is a frame for $\mathcal{S}^{\prime}$. Some of the items of Theorem 2 generalize [36, Proposition $2.2]$.

As a consequence of Theorem 2 and Theorem 1, we obtain:

Corollary 1 If $\mathcal{F}=\left\{f_{i}\right\}_{i \in I} \subseteq \mathcal{V}$ and $\Phi=\left\{\phi_{i}\right\}_{i \in I} \subseteq \mathcal{S}^{\prime}$ are dual frames in $\mathcal{V}$, then:

(i) $\mathcal{F}$ is a frame for $\mathcal{V}$ and $\Phi_{\mid \mathcal{V}}$ is a frame for $\mathcal{V}^{\prime}$

(ii) $\mathcal{V} \cap \operatorname{ker}\left(T_{\Phi}^{*}\right)=\{0\}$.

(iii) $\left(T_{\mathcal{F}}\right)_{\mid \operatorname{im}\left(T_{\Phi_{\mid \mathcal{V}}}^{*}\right)}: \operatorname{im}\left(T_{\Phi_{\mid \mathcal{V}}}^{*}\right) \rightarrow \mathcal{V}$ and $T_{\Phi_{\mid \mathcal{V}}}^{*}: \mathcal{V} \rightarrow \operatorname{im}\left(T_{\Phi_{\mid \mathcal{V}}}^{*}\right)$ are bijective and $\left.\left(\left(T_{\mathcal{F}}\right)_{\mid \operatorname{im}\left(T_{\Phi}^{*}\right.}^{*}\right)\right)^{-1}=T_{\Phi_{\mid \mathcal{V}}}^{*}$.

Example 5 Let $\mathcal{S}$ be a finite-dimensional real or complex inner product vector space. Continuing Example 4 , let $\mathcal{F}=\left\{f_{i}\right\}_{i \in I} \subset \mathcal{V}$ and $\mathcal{G}=\left\{g_{i}\right\}_{i \in I} \subset$ $\mathcal{S}$. Let $\mathcal{W}:=\operatorname{span}\left\{g_{j}\right\}_{j \in J}$. From Example 1 and Corollary 1 , it follows that if $\mathcal{F}$ and $\mathcal{G}$ are duals in $\mathcal{V}$, then $\mathcal{V} \cap \mathcal{W}^{\perp}=\{0\}$, whereas if $\mathcal{G}$ and $\mathcal{F}$ are duals in $\mathcal{W}$, then $\mathcal{W} \cap \mathcal{V}^{\perp}=\{0\}$. 
The condition $\mathcal{V} \cap \mathcal{W}^{\perp}=\mathcal{W} \cap \mathcal{V}^{\perp}=\{0\}$ is equivalent to $\mathcal{S}=\mathcal{V} \oplus \mathcal{W}^{\perp}=$ $\mathcal{W} \oplus \mathcal{V}^{\perp}$, and in this case $\operatorname{dim}(\mathcal{W})=\operatorname{dim}(\mathcal{V})$ [35].

Suppose that $\mathcal{S}=\mathcal{V} \oplus \mathcal{W}^{\perp}=\mathcal{W} \oplus \mathcal{V}^{\perp}$ and consider the oblique projections $P_{\mathcal{V}, \mathcal{W} \perp}$ and $P_{\mathcal{W}, \mathcal{V} \perp}$. By Theorem 2, $\mathcal{F}$ and $\mathcal{G}$ are duals in $\mathcal{V}$ if and only if $P_{\mathcal{V}, \mathcal{W}^{\perp}}=T_{\mathcal{F}} T_{\mathcal{G}}^{*}$, whereas $\mathcal{G}$ and $\mathcal{F}$ are duals in $\mathcal{W}$ if and only if $P_{\mathcal{W}, \mathcal{V} \perp}=$ $T_{\mathcal{G}} T_{\mathcal{F}}^{*}$. Since $P_{\mathcal{V}, \mathcal{W}^{\perp}}^{*}=P_{\mathcal{W}, \mathcal{V} \perp}, \mathcal{F}$ and $\mathcal{G}$ are duals in $\mathcal{V}$ if and only if $\mathcal{G}$ and $\mathcal{F}$ are duals in $\mathcal{W}$. We see that in this case our definition of dual frames coincide with the concept of oblique dual frames (see [15] for the definition of oblique dual frame).

By Corollary 1, if $\mathcal{F}=\left\{f_{i}\right\}_{i \in I} \subseteq \mathcal{V}$ and $\Phi=\left\{\phi_{i}\right\}_{i \in I} \subseteq \mathcal{S}^{\prime}$ are dual frames in $\mathcal{V}$, then $\mathcal{V} \oplus \operatorname{ker}\left(T_{\Phi}^{*}\right) \subseteq \mathcal{S}$. In Remark 5 , we will see that there exist pairs of dual frames such that $\mathcal{S} \neq \mathcal{V} \oplus \operatorname{ker}\left(T_{\Phi}^{*}\right)$.

Theorem 3 Let $\mathcal{F}=\left\{f_{i}\right\}_{i \in I} \subseteq \mathcal{V}$ and $\Phi=\left\{\phi_{i}\right\}_{i \in I} \subseteq \mathcal{S}^{\prime}$. If $\mathcal{S}=\mathcal{V} \oplus$ $\operatorname{ker}\left(T_{\Phi}^{*}\right)$, then the following conditions are equivalent:

(i) $\mathcal{F}$ and $\Phi$ are dual in $\mathcal{V}$.

(ii) $i m\left(T_{\mathcal{F}}\right)=\mathcal{V}, \mathbb{F}^{I}=\operatorname{im}\left(T_{\Phi}^{*}\right) \oplus \operatorname{dep}(\mathcal{F})$ and $T_{\Phi}^{*} T_{\mathcal{F}}=P_{\operatorname{im}\left(T_{\Phi}^{*}\right), \operatorname{dep}(\mathcal{F})}$.

(iii) $T_{\mathcal{F}} T_{\Phi}^{*}=P_{\mathcal{V}, \operatorname{ker}\left(T_{\Phi}^{*}\right)}$.

Proof. If $\mathcal{S}=\mathcal{V} \oplus \operatorname{ker}\left(T_{\Phi}^{*}\right)$, then $\operatorname{im}\left(T_{\Phi}^{*}{ }_{\mathcal{V}}\right)=\operatorname{im}\left(T_{\Phi}^{*}\right)$. Thus, the equivalence of (i) and (ii) follows from Theorem 2 .

(ii) $\Rightarrow$ (iii). Suppose that (ii) holds. Then $\operatorname{im}\left(T_{\mathcal{F}} T_{\Phi}^{*}\right)=\mathcal{V}$. If $f \in$ $\operatorname{ker}\left(T_{\mathcal{F}} T_{\Phi}^{*}\right)$, then

$$
T_{\Phi}^{*} f \in \operatorname{dep}(\mathcal{F}) \cap \operatorname{im}\left(T_{\Phi}^{*}\right)
$$

and, consequently, $T_{\Phi}^{*} f=0$. Hence, $\operatorname{ker}\left(T_{\mathcal{F}} T_{\Phi}^{*}\right)=\operatorname{ker}\left(T_{\Phi}^{*}\right)$. Now

$$
\left(T_{\mathcal{F}} T_{\Phi}^{*}\right)^{2}=T_{\mathcal{F}} T_{\Phi}^{*} T_{\mathcal{F}} T_{\Phi}^{*}=T_{\mathcal{F}} P_{\operatorname{im}\left(T_{\Phi}^{*}\right), \operatorname{dep}(\mathcal{F})} T_{\Phi}^{*}=T_{\mathcal{F}} T_{\Phi}^{*}
$$

Therefore, $T_{\mathcal{F}} T_{\Phi}^{*}=P_{\mathcal{V}, \operatorname{ker}\left(T_{\Phi}^{*}\right)}$.

(iii) $\Rightarrow$ (i). It is straightforward.

Let $\mathcal{F}$ and $\mathcal{G}$ be finite subset of vectors in $\mathcal{V}$ such that $\operatorname{dep}(\mathcal{F})=\operatorname{dep}(\mathcal{G})$. Clearly, if $\left(T_{\mathcal{F}}\right)_{\mid \operatorname{dep}(\mathcal{F})^{c}}=\left(T_{\mathcal{G}}\right)_{\mid \operatorname{dep}(\mathcal{F})^{c}}$, where $\operatorname{dep}(\mathcal{F})^{c}$ is a complement of $\operatorname{dep}(\mathcal{F})$, then $\mathcal{F}=\mathcal{G}$. Thus, by Theorem 3 , we have:

Corollary 2 Let $\mathcal{F}=\left\{f_{i}\right\}_{i \in I}$ and $\mathcal{G}=\left\{g_{i}\right\}_{i \in I}$ be frames for $\mathcal{V}$. If $\mathcal{S}=$ $\mathcal{V} \oplus \operatorname{ker}\left(T_{\Phi}^{*}\right)$ and there exists $\Phi=\left\{\phi_{i}\right\}_{i \in I} \subseteq \mathcal{S}^{\prime}$ such that $\Phi$ is a dual of $\mathcal{F}$ and $\mathcal{G}$ in $\mathcal{V}$, then $\mathcal{F}=\mathcal{G}$

As another consequence of Theorem 2 we obtain: 
Corollary 3 Let $\mathcal{F}=\left\{f_{i}\right\}_{i \in I} \subseteq \mathcal{V}$ and $\Phi=\left\{\phi_{i}\right\}_{i \in I} \subseteq \mathcal{S}^{\prime}$. Then the following statements are equivalent:

(i) $\mathcal{F}$ and $\Phi$ are dual in $\mathcal{V}$.

(ii) $\operatorname{im}\left(T_{\Phi}\right)=\mathcal{V}^{\prime},\left(T_{\widehat{\mathcal{F}}}^{*}\right)_{\mid \mathcal{V}^{\prime}}$ is injective and $\left(T_{\widehat{\mathcal{F}}}^{*} T_{\Phi}\right)^{2}=T_{\widehat{\mathcal{F}}}^{*} T_{\Phi}$.

(iii) $\operatorname{im}\left(T_{\Phi}\right)=\mathcal{V}^{\prime}$ and $T_{\Phi}\left(T_{\widehat{\mathcal{F}}}^{*} T_{\Phi}\right)=T_{\Phi}$.

(iv) $\left(T_{\widehat{\mathcal{F}}}^{*}\right)_{\mid \mathcal{V}^{\prime}}$ is injective and $\left(T_{\widehat{\mathcal{F}}}^{*} T_{\Phi}\right)\left(T_{\widehat{\mathcal{F}}}^{*}\right)_{\mid \mathcal{V}^{\prime}}=\left(T_{\widehat{\mathcal{F}}}^{*}\right)_{\mid \mathcal{V}^{\prime}}$.

(v) $\operatorname{im}\left(T_{\Phi}\right)=\mathcal{V}^{\prime}, \mathbb{F}^{I}=\operatorname{im}\left(\left(T_{\widehat{\mathcal{F}}}^{*}\right)_{\mid \mathcal{V}^{\prime}}\right) \oplus \operatorname{ker}\left(T_{\Phi}\right)$ and $T_{\widehat{\mathcal{F}}}^{*} T_{\Phi}=P_{\operatorname{im}\left(\left(T_{\mathcal{\mathcal { F }}}^{*}\right)_{\mid \mathcal{V}^{\prime}}\right), \operatorname{ker}\left(T_{\Phi}\right)}$.

(vi) $\operatorname{im}\left(T_{\Phi}\right)=\mathcal{V}^{\prime},\left(T_{\widehat{\mathcal{F}}}^{*}\right)_{\mid \mathcal{V}^{\prime}}$ is injective and the matrix $\left(\phi_{j}\left(f_{i}\right)\right)_{i, j \in I}$ has eigenvector $\left(\phi_{j}\left(f_{i}\right)\right)_{i \in I}$ with eigenvalue 1 for each $j \in I$ such that $\phi_{j} \neq 0$.

Remark 4 The matrix of $T_{\widehat{\mathcal{F}}}^{*} T_{\Phi}$ with respect to the standard basis of $\mathbb{F}^{I}$ is $\left(\phi_{j}\left(f_{i}\right)\right)_{i \in I, j \in I}$, the transpose of the matrix $\left(\phi_{j}\left(f_{i}\right)\right)_{j \in I, i \in I}$ corresponding to $T_{\Phi}^{*} T_{\mathcal{F}}$.

The following theorem characterizes dual frames in terms of dual independent sets. It can be viewed as analogous to [14, Theorems 8.3.2 and 8.3.3] about $R$-duals in Hilbert spaces.

Theorem 4 Let $\left\{v_{k}\right\}_{k=1}^{d}$ be a basis for $\mathcal{V}$ and $\left\{v_{k}^{\prime}\right\}_{k=1}^{d}$ be the basis for $\mathcal{V}^{\prime}$ dual to $\left\{v_{k}\right\}_{k=1}^{d}$. Let $\mathcal{R}$ be any vector space over $\mathbb{F}$ such that $\operatorname{dim}(\mathcal{R})>$ $|I|$. Let $\left\{e_{i}\right\}_{i \in I} \subseteq \mathcal{R}$ be linearly independent and $\left\{e_{i}^{\prime}\right\}_{i \in I} \subseteq \mathcal{R}^{\prime}$ be such that $e_{i}^{\prime}\left(e_{j}\right)=\delta_{i, j}$. Let $\mathcal{F}=\left\{f_{i}\right\}_{i \in I} \subseteq \mathcal{V}, \Phi=\left\{\phi_{i}\right\}_{i \in I} \subseteq \mathcal{S}^{\prime}, \omega_{k}=\sum_{i \in I} v_{k}^{\prime}\left(f_{i}\right) e_{i}$ and $\gamma_{k}=\sum_{i \in I} \phi_{i}\left(v_{k}\right) e_{i}^{\prime}$ for each $k=1, \ldots, d$. Then:

(i) $\mathcal{F}$ is a frame for $\mathcal{V}$ if and only if $\left\{\omega_{k}\right\}_{k=1}^{d}$ is linearly independent.

(ii) $(\Phi)_{\mid \mathcal{V}}$ is a frame for $\mathcal{V}^{\prime}$ if and only if $\left\{\gamma_{k}\right\}_{k=1}^{d}$ is linearly independent.

(iii) $\mathcal{F}$ and $\Phi$ are dual frames in $\mathcal{V}$ if and only if $\gamma_{l}\left(\omega_{k}\right)=\delta_{k, l}$ for each $k, l=1, \ldots, d$.

Proof. (i). Let $c \in \mathbb{F}^{d}$. We have

$$
\sum_{k=1}^{d} c(k) \omega_{k}=\sum_{i \in I}\left[\sum_{k=1}^{d} c(k) v_{k}^{\prime}\left(f_{i}\right)\right] e_{i} .
$$

Since $\left\{e_{i}\right\}_{i \in I}$ is linearly independent, $\sum_{k=1}^{d} c(k) \omega_{k}=0$ if and only if

$$
\sum_{k=1}^{d} c(k) v_{k}^{\prime}\left(f_{i}\right)=0 \quad \text { for each } i \in I .
$$


If $\mathcal{F}$ is a frame for $\mathcal{V}$, this implies that $\sum_{k=1}^{d} c(k) v_{k}^{\prime}=0$. Now using that $\left\{v_{k}^{\prime}\right\}_{k=1}^{d}$ is linearly independent, we obtain

$$
c(k)=0 \quad \text { for each } k=1, \ldots, d .
$$

This shows that $\left\{\omega_{k}\right\}_{k=1}^{d}$ is linearly independent.

Conversely, assume that $\left\{\omega_{k}\right\}_{k=1}^{d}$ is linearly independent. Let $\lambda \in \mathcal{V}^{\prime}$ and suppose that

$$
\widehat{f_{i}}(\lambda)=0 \quad \text { for each } i \in I .
$$

Since $\left\{v_{k}^{\prime}\right\}_{k=1}^{d}$ is a basis for $\mathcal{V}^{\prime}$, there exists $c \in \mathbb{F}^{d}$ such that $\lambda=\sum_{k=1}^{d} c(k) v_{k}^{\prime}$. Then

$$
\sum_{k=1}^{d} c(k) v_{k}^{\prime}\left(f_{i}\right)=0 \quad \text { for each } i \in I
$$

and, by (1),

$$
\sum_{k=1}^{d} c(k) \omega_{k}=0 .
$$

Since $\left\{\omega_{k}\right\}_{k=1}^{d}$ is linearly independent, $c(k)=0$ for each $k=1, \ldots, d$. Therefore, $\lambda=0$. This shows that $T_{\mathcal{F}}^{*}$ is injective. By Proposition $1, \mathcal{F}$ is a frame for $\mathcal{V}$.

(ii). The proof is analogous to the proof of (i).

(iii). If $\mathcal{F}$ and $\Phi$ are dual in $\mathcal{V}$, then

$$
\gamma_{l}\left(\omega_{k}\right)=\sum_{i \in I} v_{k}^{\prime}\left(f_{i}\right) \phi_{i}\left(v_{l}\right)=v_{k}^{\prime}\left(\sum_{i \in I} \phi_{i}\left(v_{l}\right) f_{i}\right)=v_{k}^{\prime}\left(v_{l}\right)=\delta_{k, l}
$$

for each $k, l=1, \ldots, d$. Conversely, suppose that $\gamma_{l}\left(\omega_{k}\right)=\delta_{k, l}$ for each $k, l=1, \ldots, d$. Then

$$
v_{k}^{\prime}\left(\sum_{i \in I} \phi_{i}\left(v_{l}\right) f_{i}\right)=\gamma_{l}\left(\omega_{k}\right)=v_{k}^{\prime}\left(v_{l}\right) \quad \text { for each } k, l=1, \ldots, d
$$

Since $\left\{v_{k}^{\prime}\right\}_{k=1}^{d}$ is a basis for $\mathcal{V}^{\prime}$,

$$
\sum_{i \in I} \phi_{i}\left(v_{l}\right) f_{i}=v_{l} \quad \text { for each } l=1, \ldots, d \text {. }
$$

Now using that $\left\{v_{k}\right\}_{k=1}^{d}$ is a basis for $\mathcal{V}$, we obtain

$$
\sum_{i \in I} \phi_{i}(f) f_{i}=f \quad \text { for each } f \in \mathcal{V} .
$$

Therefore, $\mathcal{F}$ and $\Phi$ are dual in $\mathcal{V}$. 
In the following proposition, we present the conditions under which the application of a linear transformation to a pair of dual frames results in a pair of dual frames.

Proposition 3 Let $\mathcal{R}$ be a vector space over $\mathbb{F}, \mathcal{W}$ be a subspace of $\mathcal{R}$, $A \in L(\mathcal{V}, \mathcal{W})$ and $B \in L(\mathcal{R}, \mathcal{S})$ with $B(\mathcal{W}) \subseteq \mathcal{V}$. Let $\mathcal{F}=\left\{f_{i}\right\}_{i \in I} \subseteq \mathcal{V}$ and $\Phi=\left\{\phi_{i}\right\}_{i \in I} \subseteq \mathcal{S}^{\prime}$ be dual frames in $\mathcal{V}$. Then $A \mathcal{F}=\left\{A f_{i}\right\}_{i \in I} \subseteq \mathcal{W}$ and $\Phi B=\left\{\phi_{i} B\right\}_{i \in I} \subseteq \mathcal{R}^{\prime}$ are dual frames in $\mathcal{W}$ if and only if $A B_{\mid \mathcal{W}}=I_{\mathcal{W}}$.

Proof. It is sufficient to note that $T_{A \mathcal{F}} T_{\Phi B}^{*}=A T_{\mathcal{F}} T_{\Phi}^{*} B$.

The next two results show that if we apply a linear transformation $B \in$ $L(\mathcal{S}, \mathcal{S})$ with $B(\mathcal{V}) \subseteq \mathcal{V}$ to a dual frame, we always obtain the same dual.

Proposition 4 Let $B \in L(\mathcal{S}, \mathcal{S})$ with $B(\mathcal{V}) \subseteq \mathcal{V}$. Let $\Phi, \widetilde{\Phi} \subseteq \mathcal{S}^{\prime}$ be dual frames of $\mathcal{F} \subseteq \mathcal{V}$ in $\mathcal{V}$. Then $\widetilde{\Phi}=\Phi B$ if and only if $B_{\mid \mathcal{V}}=I_{\mathcal{V}}$.

Proof. Suppose that $\widetilde{\Phi}=\Phi B$. Then

$$
\left(T_{\mathcal{F}} T_{\Phi}^{*}\right)_{\mid \mathcal{V}}=I_{\mathcal{V}}=\left(T_{\mathcal{F}} T_{\widetilde{\Phi}}^{*}\right)_{\mid \mathcal{V}}=\left(T_{\mathcal{F}} T_{\Phi}^{*} B\right)_{\mid \mathcal{V}}
$$

Since $B(\mathcal{V}) \subseteq \mathcal{V}$, it results that $B_{\mid \mathcal{V}}=I_{\mathcal{V}}$

The following proposition can be obtained with a similar proof:

Proposition 5 Let $B \in L(\mathcal{S}, \mathcal{S})$ with $B(\mathcal{V}) \subseteq \mathcal{V}$. Let $\mathcal{F}, \widetilde{\mathcal{F}} \subseteq \mathcal{V}$ be dual frames of $\Phi \subseteq \mathcal{S}^{\prime}$ in $\mathcal{V}$. Then $\widetilde{\mathcal{F}}=B \mathcal{F}$ if and only if $B_{\mid \mathcal{V}}=I_{\mathcal{V}}$.

Next we show that every pair of families in $\mathcal{V}$ and $\mathcal{S}^{\prime}$ can be extended to a pair of dual frames using an existing pair of dual frames. Concretely, in our settings, we have the following version of [14, Theorem 6.4.1.]:

Theorem 5 Let $\mathcal{F}=\left\{f_{i}\right\}_{i \in I} \subseteq \mathcal{V}$ and $\Phi=\left\{\phi_{i}\right\}_{i \in I} \subseteq \mathcal{S}^{\prime}$. If $\left\{\tilde{f}_{i}\right\}_{j \in J} \subseteq \mathcal{V}$ and $\left\{\widetilde{\phi}_{i}\right\}_{i \in I} \subseteq \mathcal{S}^{\prime}$ is any pair of dual frames in $\mathcal{V}$, then $\left\{f_{i}\right\}_{i \in I} \cup\left\{\widetilde{f}_{i}\right\}_{j \in J} \subseteq \mathcal{V}$ and $\left\{\phi_{i}\right\}_{i \in I} \cup\left\{\widetilde{\phi}_{j}-\sum_{i \in I} \widetilde{\phi}_{j}\left(f_{i}\right) \phi_{i}\right\}_{j \in J} \subseteq \mathcal{S}^{\prime}$ are dual frames in $\mathcal{V}$.

Proof. The composition of the synthesis operator of $\left\{f_{i}\right\}_{i \in I} \cup\left\{\tilde{f}_{i}\right\}_{j \in J}$ with the analysis operator of

$$
\left\{\phi_{i}\right\}_{i \in I} \cup\left\{\widetilde{\phi}_{j}-\sum_{i \in I} \widetilde{\phi}_{j}\left(f_{i}\right) \phi_{i}\right\}_{j \in J}
$$

at $f \in \mathcal{V}$ is

$$
T_{\mathcal{F}} T_{\Phi}^{*} f+T_{\widetilde{\mathcal{F}}} T_{\widetilde{\Phi}}^{*}\left(I_{\mathcal{V}}-T_{\mathcal{F}} T_{\Phi}^{*}\right) f=f .
$$

From here, the conclusion follows.

We note that a result analogous to the previous theorem can be obtained for any pair of families in $\mathcal{V}^{\prime}$ and $\mathcal{S}^{\prime \prime}$. 


\section{The construction of all dual frames of a given frame}

In this section, we prove that any frame has duals. Moreover, we show how we can obtain all duals of a given frame.

Theorem 6 Let $\mathcal{F}=\left\{f_{i}\right\}_{i \in I}$ be a frame for $\mathcal{V}$. The duals $\Phi=\left\{\phi_{i}\right\}_{i \in I} \subseteq \mathcal{S}^{\prime}$ of $\mathcal{F}$ in $\mathcal{V}$ are the families $\Phi=\left\{p_{i} B\right\}_{i \in I}$ where $B \in L\left(\mathcal{S}, \mathbb{F}^{I}\right)$ and $B_{\mid \mathcal{V}}$ is a right inverse of $T_{\mathcal{F}}$. In this case, $B=T_{\Phi}^{*}$.

Proof. Let $B \in L\left(\mathcal{S}, \mathbb{F}^{I}\right)$ be any extension of a linear right inverse of $T_{\mathcal{F}}$. Set $\phi_{i}:=p_{i} B \in \mathcal{S}^{\prime}$ for each $i \in I$ and $\Phi=\left\{\phi_{i}\right\}_{i \in I}$. Then $T_{\Phi}^{*}=B$ is injective and $\Phi_{\mid \mathcal{V}}$ is a frame for $\mathcal{V}^{\prime}$. Since

$$
T_{\mathcal{F}} T_{\Phi}^{*} f=T_{\mathcal{F}} B f=f \quad \text { for each } f \in \mathcal{V},
$$

$\mathcal{F}$ and $\Phi$ are dual frames in $\mathcal{V}$.

Conversely, let $\Phi=\left\{\phi_{i}\right\}_{i \in I} \subseteq \mathcal{S}^{\prime}$ be a dual of $\mathcal{F}$. Then $T_{\Phi_{\mid V}}^{*}$ is a linear right inverse of $T_{\mathcal{F}}$ and $\phi_{i}=p_{i} T_{\Phi}^{*}$ for each $i \in I$.

Remark 5 In the previous theorem, $T_{\Phi}^{*}$ is any extension of a linear right inverse of $T_{\mathcal{F}}$; therefore we can have $T_{\Phi}^{*}$ such that $\mathcal{S} \neq \mathcal{V} \oplus \operatorname{ker}\left(T_{\Phi}^{*}\right)$.

Example 6 We continue Example 4 . The frame operator of $\mathcal{F}=\left\{f_{i}\right\}_{i \in I} \subset \mathcal{V}$ is

$$
S_{\mathcal{F}}:=T_{\mathcal{F}} T_{\mathcal{F}}^{*} \in L(\mathcal{S}) .
$$

If $\mathcal{F}$ is a frame for $\mathcal{V}$, the family

$$
\left(S_{\mathcal{F}}\right)_{\mathcal{V}, \mathcal{V}^{\perp}}^{\sharp} \mathcal{F}=\left\{\left(S_{\mathcal{F}}\right)_{\mathcal{V}, \mathcal{V}^{\perp}}^{\sharp} f_{i}\right\}_{i \in I}
$$

is called the canonical dual frame of $\mathcal{F}$ in $\mathcal{V}$. If $\widetilde{f}_{i}=\left(S_{\mathcal{F}}\right)_{\mathcal{V}, \mathcal{V}}^{\sharp} f_{i}$ for each $i \in I$, then

$$
T_{\widetilde{\mathcal{F}}}=\left(S_{\mathcal{F}}\right)_{\mathcal{V}, \mathcal{V}^{\perp}}^{\sharp} T_{\mathcal{F}} \quad \text { and } \quad T_{\mathcal{F}} T_{\widetilde{\mathcal{F}}}^{*}=P_{\mathcal{V}, \mathcal{V}^{\perp}} .
$$

Thus, $T_{\widetilde{\mathcal{F}}}^{*} \in L\left(\mathcal{S}, \mathbb{F}^{I}\right)$ is such that $\left(T_{\widetilde{\mathcal{F}}}^{*}\right)_{\mathcal{V}}$ is a right inverse of $T_{\mathcal{F}}$. In this case $\operatorname{ker}\left(T_{\widetilde{\mathcal{F}}}^{*}\right)=\mathcal{V}^{\perp}$.

The following two corollaries give explicit expressions of all duals of a given frame starting from a given dual.

Corollary 4 Let $\mathcal{F}=\left\{f_{i}\right\}_{i \in I}$ be a frame for $\mathcal{V}$ and $\Phi=\left\{\phi_{i}\right\}_{i \in I} \subseteq \mathcal{S}^{\prime}$ be a dual of $\mathcal{F}$ in $\mathcal{V}$. Then the duals of $\mathcal{F}$ in $\mathcal{V}$ are the families $\left\{\phi_{i}-\lambda_{i}+\right.$ $\left.\sum_{j \in I} \phi_{i}\left(f_{j}\right) \lambda_{j}\right\}_{i \in I}$ where $\left\{\lambda_{i}\right\}_{i \in I} \subseteq \mathcal{S}^{\prime}$. 
Proof. Let $B=T_{\Phi}^{*} \in L\left(\mathcal{S}, \mathbb{F}^{I}\right)$. Then $B_{\mathcal{V}}$ is a right inverse of $T_{\mathcal{F}}$ and $\phi_{i}=p_{i} B$ for each $i \in I$. Any $\widetilde{B} \in L\left(\mathcal{S}, \mathbb{F}^{I}\right)$ such that $\widetilde{B}_{\mid \mathcal{V}}$ is a right inverse of $T_{\mathcal{F}}$ is of the form

$$
\widetilde{B}=B+\left(B T_{\mathcal{F}}-I_{\mathbb{F}^{I}}\right) \Lambda
$$

where $\Lambda \in L\left(\mathcal{S}, \mathbb{F}^{I}\right)$. Then

$$
\left\{p_{i} \widetilde{B}\right\}_{i \in I}=\left\{\phi_{i}-\lambda_{i}+\sum_{j \in I} \phi_{i}\left(f_{j}\right) \lambda_{j}\right\}_{i \in I},
$$

where $\lambda_{i}=p_{i} \Lambda \in \mathcal{S}^{\prime}$ for each $i \in I$. Now the conclusion follows from Theorem 6 .

Corollary 5 Let $\mathcal{F}=\left\{f_{i}\right\}_{i \in I}$ be a frame for $\mathcal{V}$ and $\Phi=\left\{\phi_{i}\right\}_{i \in I} \subseteq \mathcal{S}^{\prime}$ be a dual of $\mathcal{F}$ in $\mathcal{V}$. Then the duals of $\mathcal{F}$ in $\mathcal{V}$ are the families $\left\{\phi_{i}+\lambda_{i}\right\}_{i \in I}$ where $\left\{\lambda_{i}\right\}_{i \in I} \subseteq \mathcal{S}^{\prime}$ is such that $\sum_{i \in I} \lambda_{i}\left(f_{j}\right) f_{i}=0$ for each $j \in I$.

Proof. Let $B$ be as in the previous proof. Any $\widetilde{B} \in L\left(\mathcal{S}, \mathbb{F}^{I}\right)$ such that $\widetilde{B}_{\mid \mathcal{V}}$ is a right inverse of $T_{\mathcal{F}}$ is of the form

$$
\widetilde{B}=B+\Lambda
$$

where $\Lambda \in L\left(\mathcal{S}, \mathbb{F}^{I}\right)$ is such that $T_{\mathcal{F}} \Lambda_{\mid \mathcal{V}}=0$. Applying Theorem 6 , we obtain the conclusion.

Taking into account the proof of Corollary 5, we conclude that if $\mathcal{V}=\mathcal{S}$ and $\operatorname{dim}(\mathcal{V})=d$, then the affine space of the duals of a frame $\mathcal{F}$ for $\mathcal{V}$ has dimension

$$
d(|I|-d)=\operatorname{dim}(L(\mathcal{V}, \operatorname{dep}(\mathcal{F}))) .
$$

This affine space reduces to a single point if and only if $|I|=d$.

Example 7 We continue Example 6. Let $\mathcal{F}=\left\{f_{i}\right\}_{i \in I}$ be a frame for $\mathcal{V}$ and let $\widetilde{\mathcal{F}}=\left\{\widetilde{f}_{i}\right\}_{i \in I}$ be the canonical dual frame of $\mathcal{F}$ in $\mathcal{V}$. By Corollary 4 , any dual frame of $\mathcal{F}$ in $\mathcal{V}$ is of the form

$$
\left\{\widetilde{f}_{i}-h_{i}+\sum_{j \in I}\left\langle f_{j}, \widetilde{f}_{i}\right\rangle h_{j}\right\}_{i \in I}
$$

where $\left\{h_{i}\right\}_{i \in I} \subset \mathcal{S}$. From Corollary 5, any dual frame of $\mathcal{F}$ in $\mathcal{V}$ is of the form

$$
\left\{\tilde{f}_{i}+h_{i}\right\}_{i \in I},
$$

where $\left\{h_{i}\right\}_{i \in I} \subset \mathcal{S}$ is such that $\sum_{i \in I}\left\langle f_{j}, h_{i}\right\rangle f_{i}=0$ for each $j \in I$. Thus, we recover well known expressions for the duals in classical frame theory for Hilbert spaces. 
In the rest of this section, we consider similar results for the duals $\mathcal{F}=$ $\left\{f_{i}\right\}_{i \in I} \subseteq \mathcal{V}$ of $\Phi=\left\{\phi_{i}\right\}_{i \in I} \subseteq \mathcal{S}^{\prime}$ in $\mathcal{V}$.

Theorem 7 Let $\Phi=\left\{\phi_{i}\right\}_{i \in I} \subseteq \mathcal{S}^{\prime}$ be such that $\Phi_{\mid \mathcal{V}}$ is a frame for $\mathcal{V}^{\prime}$. The duals of $\Phi$ in $\mathcal{V}$ are precisely the families $\mathcal{F}=\left\{A \delta_{i}\right\}_{i \in I} \subseteq \mathcal{V}$, where $A \in L\left(\mathbb{F}^{I}, \mathcal{V}\right)$ is a left inverse of $\left(T_{\Phi}^{*}\right)_{\mid \mathcal{V}}$. In this case, $A=T_{\mathcal{F}}$.

Proof. Let $A \in L\left(\mathbb{F}^{I}, \mathcal{V}\right)$ be a left inverse of $\left(T_{\Phi}^{*}\right)_{\mid \mathcal{V}}$. Set $f_{i}:=A \delta_{i}$ for each $i \in I$ and $\mathcal{F}=\left\{f_{i}\right\}_{i \in I}$. Then $T_{\mathcal{F}}=A$ is surjective, consequently, $\left\{f_{i}\right\}_{i \in I}$ is a frame for $\mathcal{V}$. Since

$$
T_{\mathcal{F}}\left(T_{\Phi}^{*}\right)_{\mid \mathcal{V}}=A\left(T_{\Phi}^{*}\right)_{\mid \mathcal{V}}=I_{\mathcal{V}}
$$

$\mathcal{F}$ and $\Phi$ are dual frames in $\mathcal{V}$.

Conversely, let $\mathcal{F}=\left\{f_{i}\right\}_{i \in I}$ be a dual of $\Phi$ in $\mathcal{V}$. Then $T_{\mathcal{F}} \in L\left(\mathbb{F}^{I}, \mathcal{V}\right)$ is a left inverse of $\left(T_{\Phi}^{*}\right)_{\mid \mathcal{V}}$ and $f_{i}=T_{\mathcal{F}} \delta_{i}$ for each $i \in I$.

If $A \in L\left(\mathbb{F}^{I}, \mathcal{V}\right)$ is a left inverse of $\left(T_{\Phi}^{*}\right)_{\mid \mathcal{V}}$, then any left inverse $\widetilde{A} \in$ $L\left(\mathbb{F}^{I}, \mathcal{V}\right)$ of $\left(T_{\Phi}^{*}\right)_{\mid \mathcal{V}}$ is of the form

$$
\widetilde{A}=A+\Lambda\left(I_{\mathbb{F}^{I}}-T_{\Phi}^{*} A\right)
$$

where $\Lambda \in L\left(\mathbb{F}^{I}, \mathcal{V}\right)$, or

$$
\widetilde{A}=A+\Lambda,
$$

$\Lambda \in L\left(\mathbb{F}^{I}, \mathcal{V}\right)$ is such that $\Lambda\left(T_{\Phi}^{*}\right)_{\mid \mathcal{V}}=0$. Thus, analogous to Corollary 4 and Corollary 5, we obtain the following corollaries as a consequence of Theorem 7 :

Corollary 6 Let $\Phi=\left\{\phi_{i}\right\}_{i \in I} \subseteq \mathcal{S}^{\prime}$ be such that $\Phi_{\mid \mathcal{V}}$ is a frame for $\mathcal{V}^{\prime}$, and $\mathcal{F}=\left\{f_{i}\right\}_{i \in I} \subseteq \mathcal{V}$ be a dual of $\Phi$ in $\mathcal{V}$. Then the duals of $\Phi$ in $\mathcal{V}$ are the families $\left\{f_{i}+h_{i}-\sum_{j \in I} \phi_{j}\left(f_{i}\right) h_{j}\right\}_{i \in I} \subseteq \mathcal{V}$, where $\left\{h_{i}\right\}_{i \in I} \subseteq \mathcal{V}$.

Corollary 7 Let $\Phi=\left\{\phi_{i}\right\}_{i \in I} \subseteq \mathcal{S}^{\prime}$ be such that $\Phi_{\mid \mathcal{V}}$ is a frame for $\mathcal{V}^{\prime}$, and $\mathcal{F}=\left\{f_{i}\right\}_{i \in I} \subseteq \mathcal{V}$ be a dual of $\Phi$ in $\mathcal{V}$. Then the duals of $\Phi$ in $\mathcal{V}$ are the families $\left\{f_{i}+h_{i}\right\}_{i \in I} \subseteq \mathcal{V}$, where $\left\{h_{i}\right\}_{i \in I} \subseteq \mathcal{V}$ is such that $\sum_{i \in I} \phi_{i}\left(f_{j}\right) h_{i}=0$ for each $j \in I$.

\section{The construction of specific dual frames}

Now we are going to show that if any frame $\mathcal{F}$ for $\mathcal{V}$, any complement $\operatorname{dep}(\mathcal{F})^{c}$ of $\operatorname{dep}(\mathcal{F})$ in $\mathbb{F}^{I}$ and any complement $\mathcal{V}^{c}$ of $\mathcal{V}$ in $\mathcal{S}$ are given, there exists a unique dual $\Phi \subseteq \mathcal{S}^{\prime}$ of $\mathcal{F}$ in $\mathcal{V}$ such that

$$
\operatorname{ker}\left(T_{\Phi}^{*}\right)=\mathcal{V}^{c} \quad \text { and } \quad \operatorname{im}\left(T_{\Phi}^{*}\right)=\operatorname{dep}(\mathcal{F})^{c} .
$$

The construction of this dual depends on a suitable selection of $B \in L\left(\mathcal{S}, \mathbb{F}^{I}\right)$ in Theorem 6 such that $B_{\mid \mathcal{V}}$ is a right inverse of $T_{\mathcal{F}}$. 
Theorem 8 Let $\mathcal{F}=\left\{f_{i}\right\}_{i \in I}$ be a frame for $\mathcal{V}$, $\operatorname{dep}(\mathcal{F})^{c}$ be any complement of $\operatorname{dep}(\mathcal{F})$ in $\mathbb{F}^{I}$ and $\mathcal{V}^{c}$ be any complement of $\mathcal{V}$ in $\mathcal{S}$. Then there exists a unique dual $\Phi \subseteq \mathcal{S}^{\prime}$ of $\mathcal{F}$ in $\mathcal{V}$ such that $\operatorname{im}\left(T_{\Phi}^{*}\right)=\operatorname{dep}(\mathcal{F})^{c}$ and $\operatorname{ker}\left(T_{\Phi}^{*}\right)=\mathcal{V}^{c}$. The dual $\Phi$ is such that $T_{\Phi}^{*}=\left(T_{\mathcal{F}}\right)_{\operatorname{dep}(\mathcal{F})^{c}, \mathcal{V}^{c}}$, where $T_{\mathcal{F}}$ is considered as an element of $L\left(\mathbb{F}^{I}, \mathcal{S}\right)$.

Proof. By definition of $\Phi$ and $\left(T_{\mathcal{F}}\right)_{\operatorname{dep}(\mathcal{F})^{c}, \mathcal{V}^{c}}^{\sharp}$,

$$
\operatorname{im}\left(T_{\Phi}^{*}\right)=\operatorname{dep}(\mathcal{F})^{c} \quad \text { and } \quad \operatorname{ker}\left(T_{\Phi}^{*}\right)=\mathcal{V}^{c}
$$

Let $f \in \mathcal{V}$. Then

$$
T_{\mathcal{F}} T_{\Phi}^{*} f=T_{\mathcal{F}}\left(T_{\mathcal{F}}\right)_{\operatorname{dep}(\mathcal{F})^{c}, \mathcal{V}^{c}}^{\sharp} f=P_{\mathcal{V}, \mathcal{V}^{c}} f=f .
$$

This shows that $\Phi$ is a dual of $\mathcal{F}$ in $\mathcal{V}$.

By Theorem 3 , if $\Phi \subseteq \mathcal{S}^{\prime}$ is a dual of $\mathcal{F}$ such that $\operatorname{im}\left(T_{\Phi}^{*}\right)=\operatorname{dep}(\mathcal{F})^{c}$ and $\operatorname{ker}\left(T_{\Phi}^{*}\right)=\mathcal{V}^{c}$, then

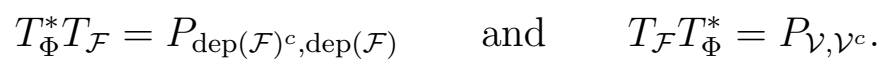

Therefore, $T_{\Phi}^{*}=\left(T_{\mathcal{F}}\right)_{\operatorname{dep}(\mathcal{F})^{c}, \mathcal{V}^{c}}^{\sharp}$. This shows the uniqueness of $\Phi$.

Example 8 Let $\mathcal{S}$ be a finite-dimensional real or complex inner product vector space. Let $\mathcal{F}=\left(f_{i}\right)_{i}$ be a frame for $\mathcal{V}$. If $\widetilde{\mathcal{F}}$ is the canonical dual frame of $\mathcal{F}$ in $\mathcal{V}$ given by $\widetilde{\mathcal{F}}=\left\{\left(S_{\mathcal{F}}\right)_{\mathcal{V}, \mathcal{V} \perp}^{\sharp} f_{i}\right\}_{i \in I}$, then

$$
T_{\mathcal{F}} T_{\widetilde{\mathcal{F}}}^{*}=P_{\mathcal{V}, \mathcal{V} \perp} \quad \text { and } \quad \operatorname{ker}\left(T_{\widetilde{\mathcal{F}}}^{*}\right)=\mathcal{V}^{\perp}
$$

(see Example 6).

Let $x \in \operatorname{dep}(\mathcal{F})^{\perp}=\operatorname{im}\left(T_{\mathcal{F}}^{*}\right)$. If $y=T_{\widetilde{\mathcal{F}}}^{*} T_{\mathcal{F}} x=T_{\mathcal{F}} x$, then

$$
T_{\mathcal{F}} y=T_{\mathcal{F}} T_{\widetilde{\mathcal{F}}}^{*} T_{\mathcal{F}} x=P_{\mathcal{V}, \mathcal{V} \perp} T_{\mathcal{F}} x=T_{\mathcal{F}} x .
$$

Thus, $x-y \in \operatorname{dep}(\mathcal{F}) \cap \operatorname{im}\left(T_{\mathcal{F}}^{*}\right)=\{0\}$. Therefore,

$$
T_{\mathcal{\mathcal { F }}}^{*} T_{\mathcal{F}} x=x, \quad T_{\mathcal{\mathcal { F }}}^{*} T_{\mathcal{F}}=P_{\operatorname{dep}(\mathcal{F})^{\perp}, \operatorname{dep}(\mathcal{F})}
$$

and

$$
\operatorname{dep}(\mathcal{F})^{\perp} \subset \operatorname{im}\left(T_{\widetilde{\mathcal{F}}}^{*}\right) .
$$

Let $x \in \operatorname{im}\left(T_{\widetilde{\mathcal{F}}}^{*}\right)$. Since $\mathcal{F}$ is a frame for $\mathcal{V}$, there exists $y \in \operatorname{dep}(\mathcal{F})^{\perp}$ such that $x=T_{\widetilde{\mathcal{F}}}^{*} T_{\mathcal{F}} y$. But

$$
T_{\mathcal{\mathcal { F }}}^{*} T_{\mathcal{F}} y=P_{\operatorname{dep}(\mathcal{F})^{\perp}, \operatorname{dep}(\mathcal{F})} y=y .
$$

Hence, $x=y \in \operatorname{dep}(\mathcal{F})^{\perp}$. This shows that $\operatorname{dep}(\mathcal{F})^{\perp}=\operatorname{im}\left(T_{\widetilde{\mathcal{F}}}^{*}\right)$.

From the previous considerations it follows that

$$
T_{\widetilde{\mathcal{F}}}^{*}=\left(T_{\mathcal{F}}\right)_{\operatorname{dep}(\mathcal{F})^{\perp}, \mathcal{V}^{\perp}}^{\sharp},
$$

where $T_{\mathcal{F}}$ is considered as an element of $L\left(\mathbb{F}^{I}, \mathcal{S}\right)$. By Theorem $8, \widetilde{\mathcal{F}}$ is the unique dual of $\mathcal{F}$ in $\mathcal{V}$ such that $\operatorname{im}\left(T_{\widetilde{\mathcal{F}}}^{*}\right)=\operatorname{dep}(\mathcal{F})^{\perp}$ and $\operatorname{ker}\left(T_{\widetilde{\mathcal{F}}}^{*}\right)=\mathcal{V}^{\perp}$. 
The following theorem gives an expression of the dual frames $\Phi$ of $\mathcal{F}$ in $\mathcal{V}$ such that $\operatorname{im}\left(T_{\Phi}^{*}\right)=\operatorname{dep}(\mathcal{F})^{c}$.

Theorem 9 Let $\mathcal{F}=\left\{f_{i}\right\}_{i \in I}$ be a frame for $\mathcal{V}$ and let $\operatorname{dep}(\mathcal{F})^{c}$ be any complement of $\operatorname{dep}(\mathcal{F})$ in $\mathbb{F}^{I}$. If $\Psi=\left\{\psi_{j}\right\}_{j \in J} \subseteq \mathcal{S}^{\prime}$ is such that $\Psi_{\mid \mathcal{V}}=$ $\left\{\left(\psi_{j}\right)_{\mid \mathcal{V}}\right\}_{j \in J}$ is a frame for $\mathcal{V}^{\prime}$ and $\operatorname{im}\left(T_{\Psi_{\mid \mathcal{V}}^{*}}^{*}\right)^{c}$ is any complement of $\operatorname{im}\left(T_{\Psi_{\mid \mathcal{V}}}^{*}\right)$ in $\mathbb{F}^{J}$, then $\Phi \subseteq \mathcal{S}^{\prime}$ such that

$$
T_{\Phi}^{*}=\left(T_{\Psi}^{*} T_{\mathcal{F}}\right)_{\operatorname{dep}(\mathcal{F})^{c}, \operatorname{im}\left(T_{\Psi}^{*}{ }^{c}{ }^{c}\right.}^{\sharp} T_{\Psi}^{*}
$$

is a dual of $\mathcal{F}$ in $\mathcal{V}$ with $\operatorname{im}\left(T_{\Phi}^{*}\right)=\operatorname{dep}(\mathcal{F})^{c}$. Moreover, if $\Phi_{1} \subseteq \mathcal{S}$ is any dual of $\mathcal{F}$ in $\mathcal{V}$ such that $\operatorname{im}\left(T_{\Phi_{1}}^{*}\right)=\operatorname{dep}(\mathcal{F})^{c}$, then $\left(\Phi_{1}\right)_{\mid \mathcal{V}}=\Phi_{\mid \mathcal{V}}$.

Proof. From $(2), \operatorname{im}\left(T_{\Phi}^{*}\right) \subseteq \operatorname{dep}(\mathcal{F})^{c}$. If $c \in \mathbb{F}^{I}$,

$$
T_{\Phi}^{*} T_{\mathcal{F}} c=\left(T_{\Psi}^{*} T_{\mathcal{F}}\right)_{\operatorname{dep}(\mathcal{F})^{c}, \operatorname{im}\left(T_{\Psi}^{*}\right)^{c}}^{*} T_{\Psi}^{*} T_{\mathcal{F}} c=P_{\operatorname{dep}(\mathcal{F})^{c}, \operatorname{dep}(\mathcal{F})} c .
$$

In particular, if $c \in \operatorname{dep}(\mathcal{F})^{c}$, then $T_{\Phi}^{*} T_{\mathcal{F}} c=c$. This shows that $\operatorname{dep}(\mathcal{F})^{c}=$ $\operatorname{im}\left(T_{\Phi}^{*}\right)$.

Let $f \in \mathcal{V}$. Then there exists $a \in \mathbb{F}^{I}$ such that $f=T_{\mathcal{F}} a$. Therefore,

$$
T_{\mathcal{F}} T_{\Phi}^{*} f=T_{\mathcal{F}}\left(T_{\Psi}^{*} T_{\mathcal{F}}\right)_{\operatorname{dep}(\mathcal{F})^{c}, \operatorname{im}\left(T_{\Psi_{\mid \mathcal{V}}}^{*}{ }^{c}\right.}^{\sharp} T_{\Psi}^{*} T_{\mathcal{F}} a=T_{\mathcal{F}} P_{\operatorname{dep}(\mathcal{F})^{c}, \operatorname{dep}(\mathcal{F})} a=T_{\mathcal{F}} a=f .
$$

This proves that $\Phi$ is a dual of $\mathcal{F}$ in $\mathcal{V}$.

Let $\Phi_{1} \subseteq \mathcal{S}$ be any dual of $\mathcal{F}$ in $\mathcal{V}$ such that $\operatorname{im}\left(T_{\Phi_{1}}^{*}\right)=\operatorname{dep}(\mathcal{F})^{c}$. If $f \in \mathcal{V}$ then

$$
f=T_{\mathcal{F}} T_{\Phi}^{*} f=T_{\mathcal{F}} T_{\Phi_{1}}^{*} f .
$$

Since $T_{\mathcal{F}}$ is injective on $\operatorname{dep}(\mathcal{F})^{c}$, we get $T_{\Phi}^{*} f=T_{\Phi_{1}}^{*} f$. Thus, $\left(\Phi_{1}\right)_{\mid \mathcal{V}}=\Phi_{\mid \mathcal{V}}$.

In view of the proof of Theorem 9, we have the following important result:

Theorem 10 Let $\mathcal{F}=\left\{f_{i}\right\}_{i \in I}$ be a frame for $\mathcal{V}$ and let $\operatorname{dep}(\mathcal{F})^{c}$ be any complement of $\operatorname{dep}(\mathcal{F})$ in $\mathbb{F}^{I}$. If $\Phi \subseteq \mathcal{S}^{\prime}$ is a dual frame of $\mathcal{F}$ in $\mathcal{V}$ such that $\operatorname{im}\left(T_{\Phi}^{*}\right)=\operatorname{dep}(\mathcal{F})^{c}$ and $f \in \mathcal{V}$, then $T_{\Phi}^{*}(f)=P_{\operatorname{dep}(\mathcal{F})^{c}, \operatorname{dep}(\mathcal{F})^{a}}$, where $a$ is any element in $\mathbb{F}^{I}$ such that $f=T_{\mathcal{F}} a$.

Remark 6 Let $\mathcal{C}_{1}$ and $\mathcal{C}_{2}$ be two complements of $\operatorname{dep}(\mathcal{F})$ in $\mathbb{F}^{I}$. Let $\Phi, \Psi \subseteq$ $\mathcal{S}^{\prime}$ be dual frames of $\mathcal{F}$ in $\mathcal{V}$ such that $\operatorname{im}\left(T_{\Phi}^{*}\right)=\mathcal{C}_{1}$ and $\operatorname{im}\left(T_{\Psi}^{*}\right)=\mathcal{C}_{2}$. Note that $\mathcal{C}_{1}$ and $\mathcal{C}_{2}$ are isomorphic and

$$
T_{\Psi}^{*}=P_{\mathcal{C}_{2}, \operatorname{dep}(\mathcal{F})^{c}} T_{\Phi}^{*} .
$$

If $\mathcal{F}$ is a basis for $\mathcal{V}$, then $\mathbb{F}^{I}$ is the unique complement of $\operatorname{dep}(\mathcal{F})=\{0\}$. Thus, there is a unique dual frame of $\mathcal{F}$ in $\mathcal{V}$ which coincides with the classical dual basis. 
Example 9 We continue Example 8, By Theorem 10, for $f \in \mathcal{V}$,

$$
T_{\widetilde{\mathcal{F}}}^{*}(f)=P_{\operatorname{dep}(\mathcal{F})^{\perp}, \operatorname{dep}(\mathcal{F})} a,
$$

where $T_{\mathcal{F}} a=f$. Therefore, for each $f \in \mathcal{V}$, the coefficients $\left\{\left\langle f, \widetilde{f}_{i}\right\rangle\right\}_{i \in I}$ have minimal $\ell_{2}$-norm among all coefficients $\left\{a_{i}\right\}_{i \in I} \subset \mathbb{F}$ such that $\sum_{i \in I} a_{i} f_{i}=f$ (see also [14, Theorem 1.1.5]).

Remark 7 Using the previous results we can see the relation between duals of similar frames. Let $\mathcal{R}$ be a vector space over $\mathbb{F}, \mathcal{W}$ be a subspace of $\mathcal{R}$ such that $\operatorname{dim}(\mathcal{W})=\operatorname{dim}(\mathcal{V})$ and let $L \in L(\mathcal{V}, \mathcal{W})$ be invertible. Let $\mathcal{F}=\left\{f_{i}\right\}_{i \in I}$ be a frame for $\mathcal{V}$ and let $\operatorname{dep}(\mathcal{F})^{c}$ be any complement of $\operatorname{dep}(\mathcal{F})$ in $\mathbb{F}^{I}$. By Lemma 2 and Theorem 8 (or Theorem 9), there exist a dual frame $\Phi \subseteq \mathcal{S}^{\prime}$ of $\mathcal{F}$ in $\mathcal{V}$ and a dual frame $\Psi \subseteq \mathcal{R}^{\prime}$ of $L \mathcal{F}$ in $\mathcal{W}$ such that

$$
\operatorname{im}\left(T_{\Phi}^{*}\right)=\operatorname{im}\left(T_{\Psi}^{*}\right)=\operatorname{dep}(\mathcal{F})^{c} .
$$

As a consequence of Theorem 10 ,

$$
T_{\Psi}^{*}(L f)=T_{\Phi}^{*}(f) \quad \text { for each } f \in \mathcal{V} .
$$

Remark 8 We can generalize Remark 7 by considering $\mathcal{W}$ as a subspace of $\mathcal{R}$ such that $\operatorname{dim}(\mathcal{W}) \leq \operatorname{dim}(\mathcal{V})$ and $L \in L(\mathcal{V}, \mathcal{W})$ surjective. Let $\mathcal{F}=\left\{f_{i}\right\}_{i \in I}$ be a frame for $\mathcal{V}$. Let $\operatorname{dep}(\mathcal{F})^{c}$ be any complement of $\operatorname{dep}(\mathcal{F})$ in $\mathbb{F}^{I}$ and let $\operatorname{dep}(L \mathcal{F})^{c}$ be any complement of $\operatorname{dep}(L \mathcal{F})$ in $\mathbb{F}^{I}$. From Lemma 2 and Theorem 8 (or Theorem 9), there exist a dual frame $\Phi \subseteq \mathcal{S}^{\prime}$ of $\mathcal{F}$ in $\mathcal{V}$ and a dual frame $\Psi \subseteq \mathcal{R}^{\prime}$ of $L \mathcal{F}$ in $\mathcal{W}$ such that

$$
\operatorname{im}\left(T_{\Phi}^{*}\right)=\operatorname{dep}(\mathcal{F})^{c} \quad \text { and } \quad \operatorname{im}\left(T_{\Psi}^{*}\right)=\operatorname{dep}(L \mathcal{F})^{c} .
$$

By Theorem 10 and Remark 1 ,

$$
T_{\Psi}^{*}(L f)=P_{\operatorname{dep}(L \mathcal{F})^{c}, \operatorname{dep}(L \mathcal{F})} T_{\Phi}^{*}(f) \quad \text { for each } f \in \mathcal{V} .
$$

We present now analogous to Theorems 8 and 9 results for the duals $\mathcal{F}=\left\{f_{i}\right\}_{i \in I} \subseteq \mathcal{V}$ of $\Phi=\left\{\phi_{i}\right\}_{i \in I} \subseteq \mathcal{S}^{\prime}$ in $\mathcal{V}$.

Theorem 11 Let $\Phi=\left\{\phi_{i}\right\}_{i \in I} \subseteq \mathcal{S}^{\prime}, \operatorname{ker}\left(T_{\Phi}^{*}\right)^{c}$ be a complement of $\operatorname{ker}\left(T_{\Phi}^{*}\right)$ in $\mathcal{S}$ and $\operatorname{im}\left(T_{\Phi}^{*}\right)^{c}$ be a complement of $\operatorname{im}\left(T_{\Phi}^{*}\right)^{c}$ in $\mathbb{F}^{I}$. Then there exists a unique dual $\mathcal{F} \subseteq \mathcal{S}$ of $\Phi$ such that $\operatorname{im}\left(T_{\mathcal{F}}\right)=\operatorname{ker}\left(T_{\Phi}^{*}\right)^{c}$ and $\operatorname{dep}(\mathcal{F})=\operatorname{im}\left(T_{\Phi}^{*}\right)^{c}$. The dual $\mathcal{F}$ is given by $T_{\mathcal{F}}=\left(T_{\Phi}^{*}\right)_{\operatorname{ker}\left(T_{\Phi}^{*}\right)^{c}, \operatorname{im}\left(T_{\Phi}^{*}\right)^{c}}^{\sharp}$.

Theorem 12 Let $\Phi=\left\{\phi_{i}\right\}_{i \in I} \subseteq \mathcal{S}^{\prime}$ be such that $\Phi_{\mid \mathcal{V}}$ is a frame for $\mathcal{V}^{\prime}$ and $\operatorname{im}\left(T_{\Phi_{\mid \mathcal{V}}}^{*}\right)^{c}$ be a complement of $\operatorname{im}\left(T_{\Phi_{\mid \mathcal{V}}}^{*}\right)$ in $\mathbb{F}^{I}$. If $\mathcal{G}=\left(g_{j}\right\}_{j \in J}$ is any frame for $\mathcal{V}$ and $\operatorname{dep}(\mathcal{G})^{c}$ is any complement of $\operatorname{dep}(\mathcal{G})$ in $\mathbb{F}^{J}$, then $\mathcal{F}=\left(f_{j}\right)_{j \in J} \subseteq \mathcal{V}$ given by

$$
T_{\mathcal{F}}=T_{\mathcal{G}}\left(T_{\Phi}^{*} T_{\mathcal{G}}\right)_{\operatorname{dep}(\mathcal{G})^{c}, \operatorname{im}\left(T_{\Phi}^{*}{ }^{*}\right)^{c}}^{\sharp}
$$

is the unique dual of $\Phi$ such that $\operatorname{dep}(\mathcal{F})=\operatorname{im}\left(T_{\Phi_{\mid \mathcal{V}}}^{*}\right)^{c}$. 


\section{Perturbation and dual frames}

The study of perturbation of frames is an important topic in classical frame theory. In this section, using obtained results, we will investigate the dual frames of the perturbed frames.

Let $\mathcal{S}$ be a finite-dimensional real or complex inner product vector space. We will consider different types of perturbations: close frames, near frames and Paley-Wiener perturbations.

\subsection{Close and near frames}

Let $\mathcal{F}$ and $\mathcal{G}$ be frames for $\mathcal{V}$. Following [2], we have the next two definitions. The frame $\mathcal{G}$ is close to $\mathcal{F}$ if there exists $\lambda \geq 0$ such that

$$
\left\|T_{\mathcal{F}} c-T_{\mathcal{G}} c\right\| \leq \lambda\left\|T_{\mathcal{F}} c\right\| \text { for each } c \in \mathbb{F}^{I} .
$$

We say that $\mathcal{F}$ and $\mathcal{G}$ are near if $\mathcal{F}$ is close to $\mathcal{G}$ and $\mathcal{G}$ is close to $\mathcal{F}$.

If $\mathcal{G}$ is close to $\mathcal{F}$, then $\operatorname{dep}(\mathcal{F}) \subseteq \operatorname{dep}(\mathcal{G})$. Therefore, there exists a surjective $Q \in L(\mathcal{V}, \mathcal{W})$ such that $Q f_{i}=g_{i}$ for each $i \in I$ (Remark 1). The relation between the dual frames of $\mathcal{F}$ and $\mathcal{G}$ is described in Remark 8 .

If $\mathcal{G}$ is close to $\mathcal{F}$ with a constant $\lambda<1$, then they have the same linear dependencies and, consequently, they are similar (Lemma 2). Finally, frames $\mathcal{F}$ and $\mathcal{G}$ are near if and only if they are similar (see [35, Section 3.8] for more details). In these two cases, the relation between the dual frames of $\mathcal{F}$ and $\mathcal{G}$ is given in Remark 7 .

\subsection{Paley-Wiener perturbations}

Now we consider generalizations of Paley-Wiener perturbations. They are also valid in the case that $\mathcal{S}$ is a separable Hilbert space and $I$ is a countable set. The following theorem generalizes [14, Theorem 22.1.1].

Theorem 13 Let $\mathcal{F}=\left\{f_{i}\right\}_{i \in I}$ be a frame for $\mathcal{S}$ and let $\widetilde{\mathcal{F}}=\left\{\tilde{f}_{i}\right\}_{i \in I}$ be a dual frame of $\mathcal{F}$. Let $\mathcal{G}=\left\{g_{i}\right\}_{i \in I} \subset \mathcal{S}$, and assume that there exist constants $\lambda, \mu \geq 0$ such that $\lambda+\mu\left\|T_{\widetilde{\mathcal{F}}}\right\|<1$ and

$$
\left\|T_{\mathcal{F}} c-T_{\mathcal{G}} c\right\| \leq \lambda\left\|T_{\mathcal{F}} c\right\|+\mu\|c\| \text { for each } c \in \mathbb{F}^{I} .
$$

Then $\mathcal{G}$ is a frame for $\mathcal{S}$ with

$$
\left\|S_{\mathcal{G}}^{-1}\right\|^{-1} \geq \frac{1}{\left\|T_{\widetilde{\mathcal{F}}}\right\|^{2}}\left(1-\lambda-\mu\left\|T_{\widetilde{\mathcal{F}}}\right\|\right)^{2} \quad \text { and } \quad\left\|S_{\mathcal{G}}\right\| \leq\left\|T_{\mathcal{F}}\right\|^{2}\left(1+\lambda+\frac{\mu}{\left\|T_{\mathcal{F}}\right\|}\right)^{2} .
$$

Taking into account that $\left\|T_{\mathcal{F}}^{*}-T_{\mathcal{G}}^{*}\right\|=\left\|T_{\mathcal{F}}-T_{\mathcal{G}}\right\|$, from Theorem 13 with $\lambda=0$, we have the following generalization of [14, Corollary 22.1.5]: 
Corollary 8 Let $\mathcal{F}=\left\{f_{i}\right\}_{i \in I}$ be a frame for $\mathcal{S}$ and let $\widetilde{\mathcal{F}}=\left\{\widetilde{f}_{i}\right\}_{i \in I}$ be a dual frame of $\mathcal{F}$. Let $\mathcal{G}=\left\{g_{i}\right\}_{i \in I} \subset \mathcal{S}$, and assume that there exists a constant $\mu \geq 0$ such that $\mu\left\|T_{\widetilde{\mathcal{F}}}\right\|<1$ and

$$
\left\|T_{\mathcal{F}}^{*} f-T_{\mathcal{G}}^{*} f\right\| \leq \mu\|f\| \text { for each } f \in \mathcal{S} .
$$

Then $\mathcal{G}$ is a frame for $\mathcal{S}$ with

$$
\left\|S_{\mathcal{G}}^{-1}\right\|^{-1} \geq \frac{1}{\left\|T_{\widetilde{\mathcal{F}}}\right\|^{2}}\left(1-\mu\left\|T_{\widetilde{\mathcal{F}}}\right\|\right)^{2} \quad \text { and } \quad\left\|S_{\mathcal{G}}\right\| \leq\left\|T_{\mathcal{F}}\right\|^{2}\left(1+\frac{\mu}{\left\|T_{\mathcal{F}}\right\|}\right)^{2} .
$$

Another perturbation result, that generalizes [10, Theorem 2], is the following:

Theorem 14 Let $\mathcal{F}=\left\{f_{i}\right\}_{i \in I}$ be a frame for $\mathcal{S}$ and let $\widetilde{\mathcal{F}}=\left\{\widetilde{f}_{i}\right\}_{i \in I}$ be a dual frame of $\mathcal{F}$. Let $\mathcal{G}=\left\{g_{i}\right\}_{i \in I} \subset \mathcal{S}$, and assume that there exist constants $\lambda_{1}, \lambda_{2}, \mu \geq 0$ such that $\max \left\{\lambda_{1}+\mu\left\|T_{\widetilde{\mathcal{F}}}\right\|, \lambda_{2}\right\}<1$ and

$$
\left\|T_{\mathcal{F}} c-T_{\mathcal{G}} c\right\| \leq \lambda_{1}\left\|T_{\mathcal{F}} c\right\|+\lambda_{2}\left\|T_{\mathcal{G}} c\right\|+\mu\|c\| \text { for each } c \in \mathbb{F}^{I} .
$$

Then $\mathcal{G}$ is a frame for $\mathcal{S}$ with

$$
\left\|S_{\mathcal{G}}^{-1}\right\|^{-1} \geq \frac{1}{\left\|T_{\widetilde{\mathcal{F}}}\right\|^{2}}\left(1-\frac{\lambda_{1}+\lambda_{2}+\mu\left\|T_{\widetilde{\mathcal{F}}}\right\|}{1+\lambda_{2}}\right)^{2}
$$

and

$$
\left\|S_{\mathcal{G}}\right\| \leq\left\|T_{\mathcal{F}}\right\|^{2}\left(1+\frac{\lambda_{1}+\lambda_{2}+\frac{\mu}{\left\|T_{\mathcal{F}}\right\|}}{1-\lambda_{2}}\right)^{2}
$$

The proofs of the previous results use the same arguments that appear in the proofs of the original ones, where $\widetilde{\mathcal{F}}=S_{\mathcal{F}^{-1}} \mathcal{F}=\left\{S_{\mathcal{F}^{-1}} f_{i}\right\}_{i \in I}$ is the canonical dual frame of $\mathcal{F}$. Furthermore, it can be seen that $T_{\widetilde{\mathcal{F}}} T_{\mathcal{G}}^{*}$ is invertible.

We next enunciate [12, Theorem 2.1.], in which the operator $T_{\widetilde{\mathcal{F}}} T_{\mathcal{G}}^{*}$ is also invertible:

Theorem 15 Let $\mathcal{F}=\left\{f_{i}\right\}_{i \in I}$ be a frame for $\mathcal{S}$ and let $\widetilde{\mathcal{F}}=\left\{\tilde{f}_{i}\right\}_{i \in I}$ be a dual frame of $\mathcal{F}$. Assume that $\mathcal{G}=\left\{g_{i}\right\}_{i \in I} \subset \mathcal{S}$ satisfies

$$
\lambda:=\sum_{i \in I}\left\|f_{i}-g_{i}\right\|^{2}<\infty \quad \text { and } \quad \mu:=\sum_{i \in I}\left\|f_{i}-g_{i}\right\|\left\|\tilde{f}_{i}\right\|<1 .
$$

Then $\mathcal{G}$ is a frame for $\mathcal{S}$ with

$$
\left\|S_{\mathcal{G}}^{-1}\right\|^{-1} \geq \frac{1}{\left\|T_{\widetilde{\mathcal{F}}}\right\|^{2}}(1-\mu)^{2} \quad \text { and } \quad\left\|S_{\mathcal{G}}\right\| \leq\left\|T_{\mathcal{F}}\right\|^{2}\left(1+\frac{\sqrt{\lambda}}{\left\|T_{\mathcal{F}}\right\|}\right)^{2} .
$$


In the previous results $T_{\widetilde{\mathcal{F}}} T_{\mathcal{G}}^{*}$ is invertible. Thus,

$$
T_{\mathcal{G}} T_{\widetilde{\mathcal{F}}}^{*}\left(T_{\mathcal{G}} T_{\widetilde{\mathcal{F}}}^{*}\right)^{-1} f=f \quad \text { for each } f \in \mathcal{V} .
$$

Consequently, $\widetilde{\mathcal{G}}=\left\{\left(T_{\widetilde{\mathcal{F}}}^{*}\left(T_{\mathcal{G}} T_{\widetilde{\mathcal{F}}}^{*}\right)^{-1}\right)^{*} \delta_{i}\right\}_{i \in I}=\left\{\left(T_{\widetilde{\mathcal{F}}} T_{\mathcal{G}}^{*}\right)^{-1} \widetilde{f}_{i}\right\}_{i \in I}$ is a dual frame of $\mathcal{G}$. We have $\operatorname{im}\left(T_{\widetilde{\mathcal{G}}}^{*}\right)=\operatorname{im}\left(T_{\widetilde{\mathcal{F}}}^{*}\right)$ and $\operatorname{dep}(\widetilde{\mathcal{G}})=\operatorname{dep}(\widetilde{\mathcal{F}})$. Therefore, by Theorem 3 , $\operatorname{dep}(\mathcal{G})$ is a complement of $\operatorname{im}\left(T_{\widetilde{\mathcal{F}}}^{*}\right)$ whereas $\operatorname{im}\left(T_{\mathcal{G}}^{*}\right)$ is a complement of $\operatorname{dep}(\widetilde{\mathcal{F}})$. We note that if $\mu=0$ in Theorem 14 , then $\operatorname{dep}(\mathcal{F})=\operatorname{dep}(\mathcal{G})$, and hence $\mathcal{F}$ and $\mathcal{G}$ are similar, and the relation between their dual frames is given in Remark 7 .

\section{Applications}

In the previous sections, the developed theory of dual frames was related to the classical frame theory in Hilbert spaces. Now we apply the general theory to study some questions about dual frames in different finite-dimensional vector spaces. We consider dual frames for vector spaces over conjugate closed subfields of the complex numbers, for metric vector spaces and for ultrametric normed vector spaces over complete non-archimedean valued fields. Finally, the representation of operators using dual frames and the application to the solution of operator equations are considered.

\subsection{The case $\mathbb{F} \subseteq \mathbb{C}$ and $\overline{\mathbb{F}}=\mathbb{F}$}

In [36], frames for finite-dimensional vector spaces $\mathcal{S}$ over fields $\mathbb{F}$ such that $\mathbb{F} \subseteq \mathbb{C}$ and $\overline{\mathbb{F}}=\mathbb{F}$ were considered. In this case,

$$
\mathcal{V}=\mathcal{S} \quad \operatorname{dep}(\mathcal{F})^{c}=\operatorname{dep}(\mathcal{F})^{\perp} \quad \text { and } \quad \operatorname{im}\left(T_{\Psi}^{*}\right)^{c}=\operatorname{im}\left(T_{\Psi}^{*}\right)^{\perp}
$$

the operator $\left(T_{\Psi}^{*} T_{\mathcal{F}}\right)_{\operatorname{dep}(\mathcal{F})^{c}, \operatorname{im}\left(T_{\Psi_{\mid \mathcal{V}}}^{*}\right)^{c}}^{\sharp}$ is the Moore-Penrose pseudoinverse of $T_{\Psi}^{*} T_{\mathcal{F}}$, and Theorem 9 coincides with [36, Lemma 4.2].

The matrix $P_{\operatorname{dep}(\mathcal{F})^{\perp}, \operatorname{dep}(\mathcal{F})}$ is called the canonical Gramian of $\mathcal{F}$. Linear functionals $\Phi=\left\{\phi_{i}\right\}_{i \in I} \subseteq \mathcal{S}^{\prime}$ given by

$$
T_{\Phi}^{*}(f)=P_{\operatorname{dep}(\mathcal{F})^{\perp}, \operatorname{dep}(\mathcal{F})^{a}}
$$

where $T_{\mathcal{F}} a=f$ for each $f \in \mathcal{V}$, are called the canonical coordinates for $\mathcal{F}$ (see Theorem 10). As in Example 9, for each $f \in \mathcal{V}$, the coefficients $T_{\Phi}^{*}(f)$ have minimal $\ell_{2}$-norm among all coefficients $\left\{a_{i}\right\}_{i \in I} \subset \mathbb{F}$ such that $\sum_{i \in I} a_{i} f_{i}=f$. There is a unique inner product on $\mathcal{S}$, defined by

$$
\langle f, g\rangle_{\mathcal{F}}:=\left\langle T_{\Phi}^{*}(f), T_{\Phi}^{*}(g)\right\rangle,
$$


for which $\mathcal{F}$ is a Parseval frame, i.e.,

$$
f=\sum_{i \in I}\left\langle f, f_{i}\right\rangle_{\mathcal{F}} f_{i} \quad \text { for each } f \in \mathcal{S} \text {. }
$$

See [13, 35, 36] for more details. In particular, these works consider cyclotomic fields.

Let $\omega:=e^{i \frac{2 \pi}{n}}$ be a primitive $n$-th root of unity. The cyclotomic field $\mathbb{Q}(\omega)$ is a $\mathbb{Q}$-vector space of dimension $d=\varphi(n)$, where $\varphi$ is the Euler phi (totient) function. A natural frame (spanning sequence) for $\mathbb{Q}(\omega)$ is given by the $n$-th roots $\mathcal{F}=\left\{\omega^{k}\right\}_{k \in \mathbb{Z}_{n}}$.

Let $\mathbb{Z}_{n}^{*}$ be the group of units of $\mathbb{Z}_{n}$ (equivalent classes corresponding to the elements of $\{0,1, \ldots, n-1\}$ which are coprime with $n)$. Let

$$
\chi_{j}=\left(1, \omega^{j}, \omega^{2 j}, \ldots, \omega^{(n-1) j}\right) \quad \text { for each } j \in \mathbb{Z}_{n} .
$$

Let $\mu$ be the Möbius function

$$
\mu(n):= \begin{cases}1, & n=1 \\ (-1)^{n}, & n \text { is square free } \\ 0, & \text { otherwise }\end{cases}
$$

From [35] (see also [13, 36]),

$$
P_{\operatorname{dep}(\mathcal{F})^{\perp}, \operatorname{dep}(\mathcal{F})}=\sum_{j \in \mathbb{Z}_{n}^{*}} \chi_{j} \chi_{j}^{*}
$$

and the canonical dual $\Phi$ of $\mathcal{F}$ satisfies

$$
\phi_{k}\left(\omega^{j}\right)=\left(P_{\operatorname{dep}(\mathcal{F})^{\perp}, \operatorname{dep}(\mathcal{F})}\right)(k, j)=\frac{1}{n} \varphi(n) \mu\left(\frac{n}{\operatorname{gcd}(k-j, n)}\right) .
$$

Let $C$ be the shift operator on $\mathbb{Q}^{n}$ given by $\left(a_{1}, \ldots, a_{n}\right) \mapsto\left(a_{2}, \ldots, a_{n}, a_{1}\right)$. We have that $P_{\operatorname{dep}(\mathcal{F})^{\perp}, \operatorname{dep}\left(T_{\mathcal{F}}\right)}$ is circulant, i.e.,

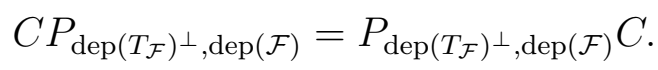

Thus, $\operatorname{im}\left(T_{\Phi}^{*}\right)=\operatorname{dep}(\mathcal{F})^{\perp}$ is invariant under the shift operator $C$. Therefore, $\operatorname{dep}(\mathcal{F})$ is shift invariant too.

Let now $\Psi=\left\{\psi_{k}\right\}_{k \in \mathbb{Z}_{n}} \subseteq \mathbb{Q}(\omega)^{\prime}$ be any dual of $\mathcal{F}$. By Theorem 2 , $\mathbb{Q}^{n}=\operatorname{im}\left(T_{\Psi}^{*}\right) \oplus \operatorname{dep}\left(T_{\mathcal{F}}\right)$. Therefore, since $\operatorname{dep}(\mathcal{F})$ is shift invariant, $\operatorname{im}\left(T_{\Psi}^{*}\right)$ is shift invariant. Let $\operatorname{im}\left(T_{\Psi}^{*}\right)=\operatorname{dep}(\mathcal{F})^{c}$, where $\operatorname{dep}\left(T_{\mathcal{F}}\right)^{c}$ is a complement of $\operatorname{dep}\left(T_{\mathcal{F}}\right)$ in $\mathbb{Q}^{n}$. If $P_{\operatorname{dep}\left(T_{\mathcal{F}}\right)^{c}, \operatorname{dep}(\mathcal{F})}$ is circulant, then $\Psi$ as the canonical dual $\Phi$ satisfies:

- Cyclic shift property: $T_{\Psi}^{*}(\omega f)=C T_{\Psi}^{*}(f)$ for each $f \in \mathbb{Q}(\omega)$. 
- Conjugate property: $T_{\Psi}^{*}(\bar{f})=C^{n} T_{\Psi}^{*}(f)$ for each $f \in \mathbb{Q}(\omega)$.

- Convolutional property: $T_{\Psi}^{*}(f g)=T_{\Psi}^{*}(f) * T_{\Psi}^{*}(g)$ for each $f, g \in \mathbb{Q}(\omega)$, where $\left[T_{\Psi}^{*}(f) * T_{\Psi}^{*}(g)\right]_{k}=\sum_{j=1}^{n-1}[\psi(f)]_{j}[\psi(g)]_{k-j}$ for each $k \in \mathbb{Z}_{n}$.

Conversely, if $\Psi$ satisfies the cyclic shift property, then $P_{\operatorname{dep}(\mathcal{F})^{c}, \operatorname{dep}(\mathcal{F})}$ is circulant. These properties are very useful for the computation of $\Psi$.

By Corollary 5 ,

$$
T_{\Psi}^{*}=T_{\Phi}^{*}+T_{\Lambda}^{*}
$$

for some $\Lambda=\left\{\lambda_{k}\right\}_{k \in \mathbb{Z}_{n}} \subseteq \mathbb{Q}(\omega)^{\prime}$ such that $\operatorname{im}\left(T_{\Lambda}^{*}\right) \subseteq \operatorname{dep}(\mathcal{F})$. Note that $\Psi$ satisfies the cyclic shift property (conjugate property) if and only if $\Lambda$ satisfies the cyclic shift property (conjugate property). We also have that if $\Lambda$ satisfies the cyclic shift property, then $\Psi$ satisfies the convolutional property. Consider the power integral basis $\mathcal{B}=\left\{\omega^{k}\right\}_{k=0}^{\varphi(n)-1}$ of $\mathbb{Q}(\omega)$ and the matrix $M_{\Lambda} \in \mathbb{Q}^{n \times n}$ such that $M_{\Lambda}(k, l):=\lambda_{k}\left(\omega^{l}\right)$. Then $\Lambda$ satisfies the cyclic shift property if and only if $M_{\Lambda}$ is circulant.

\subsection{Metric vector spaces}

Let $\mathcal{S}$ be a finite-dimensional vector space over $\mathbb{F}$ and let (.,.) be a bilinear form (bilinear functional) on $\mathcal{S}$ which is either symmetric, skew-symmetric (or antisymmetric), or alternate. The pair $(\mathcal{S},(.,)$.$) is called a metric vector$ space. If the bilinear form is symmetric, then $(\mathcal{S},(.,)$.$) is called an orthogonal$ geometry over $\mathbb{F}$, and if the bilinear form is alternate, $(\mathcal{S},(.,)$.$) is called a$ symplectic geometry over $\mathbb{F}$. For the theory of metric vector spaces see, e.g., 33.

We consider in $\mathbb{F}^{I}$ the bilinear form defined by $(x, y):=\sum_{i \in I} x(i) y(i)$ for each $x, y \in \mathbb{F}^{I}$. This bilinear form is symmetric but not alternate, and it is skew-symmetric if and only if $\operatorname{char}(\mathbb{F})=2$. The pair $\left(\mathbb{F}^{I},(\cdot, \cdot)\right)$ is a non-singular metric vector space.

Assume from now on that $\mathcal{S}$ is non-singular. Given $\phi \in \mathcal{S}^{\prime}$, there exists a unique $g \in \mathcal{S}$ such that $\phi(f)=(f, g)$ for each $f \in \mathcal{S}$ [33, Theorem 11.5]. Thus, we have a theory of dual frames for non-singular metric vector spaces as it was described in Example 1, Example 4 and Example 5 for real or complex inner product vector spaces. We note that in Example 5 the equivalence between the conditions $\mathcal{V} \cap \mathcal{W}^{\perp}=\mathcal{W} \cap \mathcal{V}^{\perp}=\{0\}$ and $\mathcal{S}=\mathcal{V} \oplus \mathcal{W}^{\perp}=\mathcal{W} \oplus \mathcal{V}^{\perp}$ can be proved as in 35 taking into account that $\operatorname{dim}(\mathcal{S})=\operatorname{dim}(\mathcal{V})+\operatorname{dim}\left(\mathcal{V}^{\perp}\right)($ see [33, Theorem 11.8]).

Now we analyze the existence of the canonical dual defined as in Example 6. First we recall some facts. Let $\mathcal{V}$ be a subspace of $\mathcal{S}$ and $\mathcal{F}=$ $\left\{f_{i}\right\}_{i \in I} \subseteq \mathcal{V}$. Then

$$
\operatorname{im}\left(T_{\mathcal{F}^{*}}\right) \perp \operatorname{ker}\left(T_{\mathcal{F}}\right), \quad \operatorname{dim}\left(\operatorname{ker}\left(T_{\mathcal{F}}\right)\right)+\operatorname{dim}\left(\operatorname{im}\left(T_{\mathcal{F}}\right)\right)=|I|
$$


and

$$
\mathbb{F}^{I} / \operatorname{ker}\left(T_{\mathcal{F}}\right) \cong \operatorname{im}\left(T_{\mathcal{F}}\right)
$$

If $\operatorname{im}\left(T_{\mathcal{F}}^{*}\right) \cap \operatorname{ker}\left(T_{\mathcal{F}}\right)=\{0\}$, then

$$
\mathbb{F}^{I} / \operatorname{ker}\left(T_{\mathcal{F}}\right) \cong \operatorname{im}\left(T_{\mathcal{F}^{*}}\right) \quad \text { and } \quad \mathbb{F}^{I}=\operatorname{ker}\left(T_{\mathcal{F}}\right) \oplus \operatorname{im}\left(T_{\mathcal{F}^{*}}\right) .
$$

Let $\mathcal{F}=\left\{f_{i}\right\}_{i \in I}$ be a frame for $\mathcal{V}$. The canonical dual of $\mathcal{F}$ (defined as in Example 6f exists if and only if $\left(S_{\mathcal{F}}\right)_{\mathcal{V}, \mathcal{V}^{\perp}}^{\sharp}$ exists, i.e.,

$$
\mathcal{S}=\mathcal{V} \oplus \mathcal{V}^{\perp}, \quad \operatorname{im}\left(S_{\mathcal{F}}\right)=\mathcal{V} \quad \text { and } \quad \operatorname{ker}\left(S_{\mathcal{F}}\right)=\mathcal{V}^{\perp}
$$

By [33, Theorem 11.8], $\mathcal{S}=\mathcal{V} \oplus \mathcal{V}^{\perp}$ if and only if $\mathcal{V}$ is non-singular. Since $\operatorname{im}\left(T_{\mathcal{F}}\right)=\mathcal{V}$ and $\operatorname{ker}\left(T_{\mathcal{F}}^{*}\right)=\mathcal{V}^{\perp}$, we always have $\operatorname{im}\left(S_{\mathcal{F}}\right) \subseteq \mathcal{V}$ and $\mathcal{V}^{\perp} \subseteq$ $\operatorname{ker}\left(S_{\mathcal{F}}\right)$.

Theorem 16 Let $\mathcal{F}=\left\{f_{i}\right\}_{i \in I}$ be a frame for $\mathcal{V}$. The canonical dual of $\mathcal{F}$ exists if and only if $\mathcal{V}$ is non-singular and $\operatorname{im}\left(T_{\mathcal{F}}^{*}\right) \cap \operatorname{ker}\left(T_{\mathcal{F}}\right)=\{0\}$.

Proof. If $\operatorname{im}\left(T_{\mathcal{F}}^{*}\right) \cap \operatorname{ker}\left(T_{\mathcal{F}}\right)=\{0\}$ then

$$
\operatorname{ker}\left(S_{\mathcal{F}}\right) \subseteq \mathcal{V}^{\perp} \quad \text { and } \quad \mathbb{F}^{I}=\operatorname{ker}\left(T_{\mathcal{F}}\right) \oplus \operatorname{im}\left(T_{\mathcal{F}}^{*}\right)
$$

Consequently, $\mathcal{V} \subseteq \operatorname{im}\left(S_{\mathcal{F}}\right)$. Thus, if $\mathcal{V}$ is non-singular and $\operatorname{im}\left(T_{\mathcal{F}}^{*}\right) \cap \operatorname{ker}\left(T_{\mathcal{F}}\right)=$ $\{0\}$, the canonical dual of $\mathcal{F}$ exists.

Suppose now that the canonical dual of $\mathcal{F}$ exists. Then $\mathcal{V}$ is non-singular, $\operatorname{im}\left(S_{\mathcal{F}}\right)=\mathcal{V}$ and $\operatorname{ker}\left(S_{\mathcal{F}}\right)=\mathcal{V}^{\perp}$. Let $c=\left(c_{i}\right)_{i \in I} \in \operatorname{im}\left(T_{\mathcal{F}}^{*}\right) \cap \operatorname{ker}\left(T_{\mathcal{F}}\right)$. Then there exists $f \in \mathcal{S}$ such that

$$
c_{i}=\left(f, f_{i}\right) \text { for each } i \in I
$$

and

$$
S_{\mathcal{F}} f=\sum_{i \in I}\left(f, f_{i}\right) f_{i}=0 .
$$

Therefore,

$$
f \in \operatorname{ker}\left(S_{\mathcal{F}}\right)=\mathcal{V}^{\perp}=\operatorname{ker}\left(T_{\mathcal{F}}^{*}\right) .
$$

Hence, $c=0$. This shows that $\operatorname{im}\left(T_{\mathcal{F}}^{*}\right) \cap \operatorname{ker}\left(T_{\mathcal{F}}\right)=\{0\}$.

Note that, if the canonical dual exists, the properties described in Example 6, Example 8, as well as the description of all duals in Example 7 are valid in this new context. When the canonical dual does not exist, other duals are needed.

In the following example the canonical dual does not exist. The frame $\mathcal{F}$ is a basis for $\mathcal{V}$ which has a unique dual frame. 
Example 10 Let $\mathcal{S}=\mathbb{Z}_{2}^{3}$ and $\mathcal{F}=\{(1,0,1),(0,1,0)\}$. We have $\operatorname{ker}\left(T_{\mathcal{F}}\right)=\{(0,0)\}, \quad \mathcal{V}=\operatorname{span}(\mathcal{F})=\{(0,0,0),(1,0,1),(0,1,0),(1,1,1)\}$ and

$$
\mathcal{V}^{\perp}=\{(0,0,0),(1,0,1)\}
$$

Since $\mathcal{V}$ is singular, the canonical dual of $\mathcal{F}$ (defined as in Example 6) does not exist. Using Theorem 8 with $\mathcal{V}^{c}=\{(0,0,0),(1,0,0)\}$, we obtain the dual

$$
\mathcal{G}=\{(0,0,1),(0,1,0)\}
$$

of $\mathcal{F}$ in $\mathcal{V}$. In this case,

$$
\operatorname{ker}\left(T_{\mathcal{G}^{*}}\right)=\mathcal{V}^{c} \quad \text { and } \quad \operatorname{im}\left(T_{\mathcal{G}^{*}}\right)=\mathbb{Z}_{2}^{2}=\operatorname{ker}\left(T_{\mathcal{F}}\right)^{c}
$$

Since $\mathcal{F}$ is a basis for $\mathcal{V}, \mathcal{G}$ is its unique dual frame.

Analogous to the Hilbert space case, we define Parseval frames.

Definition 4 Let $\mathcal{F}=\left\{f_{i}\right\}_{i \in I} \subseteq \mathcal{V}$. We say that $\mathcal{F}$ is a Parseval frame for $\mathcal{V}$ if $S_{\mathcal{F}} f=f$ for each $f \in \mathcal{V}$.

For frames and Parseval frames in the case of the metric vector space $\mathbb{Z}_{2}^{d}$, see, e. g., [7, 8, 27, 29]. Frames over finite fields in unitary and orthogonal geometries are considered in [26, 25].

The canonical dual frame of a Parseval frame always exists:

Theorem 17 If $\mathcal{F}=\left\{f_{i}\right\}_{i \in I}$ is a Parseval frame for $\mathcal{V}$, then the canonical dual $\widetilde{\mathcal{F}}$ of $\mathcal{F}$ exists and $\widetilde{\mathcal{F}}=\mathcal{F}$.

Proof. Let $\mathcal{F}=\left\{f_{i}\right\}_{i \in I}$ be a Parseval frame for $\mathcal{V}$. If $f \in \mathcal{V} \cap \mathcal{V}^{\perp}=$ $\operatorname{im}\left(T_{\mathcal{F}}\right) \cap \operatorname{ker}\left(T_{\mathcal{F}}^{*}\right)$, then

$$
f=S_{\mathcal{F}} f=T_{\mathcal{F}} T_{\mathcal{F}}^{*} f=T_{\mathcal{F}} 0=0 .
$$

This shows that $\mathcal{V}$ is non-singular. Also, we have $S_{\mathcal{F}}=P_{\mathcal{V}, \mathcal{V} \perp}$. Thus, the canonical dual $\widetilde{\mathcal{F}}=\left\{\widetilde{f}_{i}\right\}_{i \in I}$ of $\mathcal{F}$ exists and

$$
\tilde{f}_{i}=\left(S_{\mathcal{F}}\right)_{\mathcal{V}, \mathcal{V}^{\perp}}^{\sharp} f_{i}=P_{\mathcal{V}, \mathcal{V} \perp} f_{i}=f_{i}
$$

for each $i \in I$. 


\subsection{Ultrametric normed vector spaces}

Let $\mathcal{S}$ be a finite-dimensional vector space over a complete non-archimedean valued field $\mathbb{F}$. Let $\|$.$\| be an ultrametric norm on \mathcal{S}$. In what follows, we show some particularities of frames and dual frames in these type of normed vector spaces. We consider in $\mathbb{F}^{n}$ the ultrametric norm

$$
\left\|\left(x_{k}\right)_{k=1}^{n}\right\|=\max _{1 \leq k \leq n}\left|x_{k}\right|
$$

where $|$.$| is the non-archimedean valuation on \mathbb{F}$. We use the operator norm for the different operators. For details about ultrametric analysis, we refer the reader to 32 .

We begin with the following result, which is similar to [14, Proposition 1.1.2].

Proposition 6 Let $\Phi=\left\{\phi_{k}\right\}_{k=1}^{n} \subseteq \mathcal{S}^{\prime}$. Then $\Phi$ is a frame for $\mathcal{S}^{\prime}$ if and only if there exists a real $\alpha>0$ such that $\alpha\|f\| \leq\left\|T_{\Phi}^{*}(f)\right\|$ for each $f \in \mathcal{S}$.

Proof. Assume that $\Phi$ is a frame for $\mathcal{S}^{\prime}$. By Theorem $1, T_{\Phi}^{*}$ is injective. Thus, there exists $A \in L\left(\mathbb{F}^{n}, \mathcal{S}\right)$ such that $A T_{\Phi}^{*}=I_{\mathcal{S}}$. If $f \in \mathcal{S}$, then

$$
\|A\|^{-1}\|f\| \leq\left\|T_{\Phi}^{*}(f)\right\| .
$$

We can take $\alpha=\|A\|^{-1}$.

If there exists $\alpha>0$ such that $\alpha\|f\| \leq\left\|T_{\Phi}^{*}(f)\right\|$ for each $f \in \mathcal{S}$, then $T_{\Phi}^{*}$ is injective. By Theorem 1, $\Phi$ is a frame for $\mathcal{S}^{\prime}$.

The constant $\alpha$, mentioned in the previous proposition, is called a (lower) frame bound for $\Phi$. As a consequence of Proposition 6, we obtain:

Corollary 9 Let $\Phi=\left\{\phi_{k}\right\}_{k=1}^{n}$ be a frame for $\mathcal{S}^{\prime}$ with frame bound $\alpha$ and let $\beta<\alpha$. Let $\Psi=\left\{\psi_{k}\right\}_{k=1}^{n} \subseteq \mathcal{S}^{\prime}$ be such that $\left\|T_{\Psi}^{*}(f)-T_{\Phi}^{*}(f)\right\| \leq \beta\|f\|$ for each $f \in \mathcal{S}$. Then $\Psi$ is a frame for $\mathcal{S}^{\prime}$ with frame bound $\alpha-\beta$.

Another perturbation result is the following:

Corollary 10 Let $\Phi=\left\{\phi_{k}\right\}_{k=1}^{n}$ be a frame for $\mathcal{S}^{\prime}$ with frame bound $\alpha$. Let $\Psi=\left\{\psi_{k}\right\}_{k=1}^{n} \subseteq \mathcal{S}^{\prime}$ be such that $\left\|T_{\Psi}^{*}(f)-T_{\Phi}^{*}(f)\right\|<\left\|T_{\Phi}^{*}(f)\right\|$ for each $f \in \mathcal{S}$. Then $\Psi$ is a frame for $\mathcal{S}^{\prime}$ with frame bound $\alpha$.

Proof. Since $\|$.$\| is an ultrametric norm, for each f \in \mathcal{S}$, if

$$
\left\|T_{\Psi}^{*}(f)-T_{\Phi}^{*}(f)\right\|<\left\|T_{\Phi}^{*}(f)\right\|,
$$

then

$$
\left\|T_{\Psi}^{*}(f)\right\|=\left\|T_{\Phi}^{*}(f)\right\| .
$$

It remains to apply Proposition 6 . 
Let $\mathcal{F}=\left(f_{k}\right\}_{k=1}^{n} \subseteq \mathcal{S}$ and $\Phi=\left\{\phi_{k}\right\}_{k=1}^{n} \subseteq \mathcal{S}^{\prime}$. We have

$$
\left\|T_{\mathcal{F}}\right\|=\max _{1 \leq k \leq n}\left\|f_{k}\right\| \quad \text { and } \quad\left\|T_{\Phi}^{*}\right\|=\max _{1 \leq k \leq n}\left\|\phi_{k}\right\| .
$$

From these equalities and Theorem 2 we get:

Proposition 7 Let $\mathcal{F}=\left\{f_{k}\right\}_{k=1}^{n}$ be a frame for $\mathcal{S}$ and $\Phi=\left\{\phi_{k}\right\}_{k=1}^{n} \subseteq \mathcal{S}^{\prime}$ be a dual frame of $\mathcal{F}$. Then

(i) $\left\|T_{\Phi}^{*}(f)\right\| \geq\|f\|\left\|T_{\mathcal{F}}\right\|^{-1}$ for each $f \in \mathcal{S}$.

(ii) $1 \leq\left\|T_{\Phi}^{*} T_{\mathcal{F}}\right\| \leq \max _{1 \leq k \leq n}\left\|f_{k}\right\| \max _{1 \leq k \leq n}\left\|\phi_{k}\right\|$.

The following results are about the perturbation of a dual frame.

Theorem 18 Let $\mathcal{F}=\left\{f_{k}\right\}_{k=1}^{n} \subseteq \mathcal{S}$ and $\Phi=\left\{\phi_{k}\right\}_{k=1}^{n} \subseteq \mathcal{S}^{\prime}$ be dual frames and $\mathcal{G}=\left\{g_{k}\right\}_{k=1}^{n} \subseteq \mathcal{S}$. If $\max _{1 \leq k \leq n}\left\|g_{k}-f_{k}\right\|<\frac{1}{\max _{1 \leq k \leq n}\left\|\phi_{k}\right\|}$, then:

(i) $\mathcal{G}$ is a frame for $\mathcal{S}$.

(ii) The family $\Psi$ such that $T_{\Psi}^{*}=T_{\Phi}^{*}\left(T_{\mathcal{G}} T_{\Phi}^{*}\right)^{-1}$ is a dual frame of $\mathcal{G}$.

(iii) $\operatorname{im}\left(T_{\Psi}^{*}\right)=\operatorname{im}\left(T_{\Phi}^{*}\right)$ and $\operatorname{dep}(\mathcal{G})=\operatorname{dep}(\mathcal{F})$.

(iv) $\max _{1 \leq k \leq n}\left\|\psi_{k}-\phi_{k}\right\|<\max _{1 \leq k \leq n}\left\|\phi_{k}\right\|$.

Proof. We have,

$$
\left\|I_{\mathcal{S}}-T_{\mathcal{G}} T_{\Phi}^{*}\right\|=\left\|T_{\mathcal{F}} T_{\Phi}^{*}-T_{\mathcal{G}} T_{\Phi}^{*}\right\| \leq \max _{1 \leq k \leq n}\left\|g_{k}-f_{k}\right\| \max _{1 \leq k \leq n}\left\|\phi_{k}\right\|<1 .
$$

By Neumann's theorem, $T_{\mathcal{G}} T_{\Phi}^{*}$ is invertible and

$$
\left(T_{\mathcal{G}} T_{\Phi}^{*}\right)^{-1}=\sum_{j=1}^{\infty}\left(I_{\mathcal{S}}-T_{\mathcal{G}} T_{\Phi}^{*}\right)^{j} .
$$

Since $\mathcal{S}$ is an ultrametric normed vector space, from the last equality we obtain $\left\|\left(T_{\mathcal{G}} T_{\Phi}^{*}\right)^{-1}\right\|<1$.

Since

$$
f=\left(T_{\mathcal{G}} T_{\Phi}^{*}\right)\left(T_{\mathcal{G}} T_{\Phi}^{*}\right)^{-1} f \quad \text { for each } f \in \mathcal{S},
$$

one has (i) and (ii). Part (iii) is a consequence of (ii) and Theorem2, Finally,

$$
\begin{aligned}
\max _{1 \leq k \leq n}\left\|\psi_{k}-\phi_{k}\right\| & =\left\|T_{\Psi}^{*}-T_{\Phi}^{*}\right\|=\left\|T_{\Phi}^{*}\left(\left(T_{\mathcal{G}} T_{\Phi}^{*}\right)^{-1}-I_{\mathcal{S}}\right)\right\| \\
& \leq \max _{1 \leq k \leq n}\left\|\phi_{k}\right\|\left\|\left(T_{\mathcal{G}} T_{\Phi}^{*}\right)^{-1}-I_{\mathcal{S}}\right\| \\
& \leq \max _{1 \leq k \leq n}\left\|\phi_{k}\right\|\left\|\left(T_{\mathcal{G}} T_{\Phi}^{*}\right)^{-1}\right\|\left\|I_{\mathcal{S}}-T_{\mathcal{G}} T_{\Phi}^{*}\right\| \\
& <\max _{1 \leq k \leq n}\left\|\phi_{k}\right\|,
\end{aligned}
$$

which proves (iv). 
Note that by Theorem 8 (or Theorem 9), $\Psi$ in Theorem 18 is the unique dual of $\mathcal{G}$ such that $\operatorname{im}\left(T_{\Psi}^{*}\right)=\operatorname{im}\left(T_{\Phi}^{*}\right)$.

With a similar proof we get:

Theorem 19 Let $\mathcal{F}=\left\{f_{k}\right\}_{k=1}^{n}$ be a frame for $\mathcal{S}, \Phi=\left\{\phi_{k}\right\}_{k=1}^{n} \subseteq \mathcal{S}^{\prime}$ be a dual frame of $\mathcal{F}$ and $\Psi=\left\{\psi_{k}\right\}_{k=1}^{n} \subseteq \mathcal{S}^{\prime}$. If $\max _{1 \leq k \leq n}\left\|\psi_{k}-\phi_{k}\right\|<\frac{1}{\max _{1 \leq k \leq n}\left\|f_{k}\right\|}$, then:

(i) $\Psi$ is a frame for $\mathcal{S}^{\prime}$.

(ii) The family $\mathcal{G}$ such that $T_{\mathcal{G}}=\left(T_{\mathcal{F}} T_{\Psi}^{*}\right)^{-1} T_{\mathcal{F}}$ is a dual frame of $\Psi$.

(iii) $\operatorname{dep}(\mathcal{G})=\operatorname{dep}(\mathcal{F})$ and $\operatorname{im}\left(T_{\Psi}^{*}\right)=\operatorname{im}\left(T_{\Phi}^{*}\right)$.

(iv) $\max _{1 \leq k \leq n}\left\|g_{k}-f_{k}\right\|<\max _{1 \leq k \leq n}\left\|f_{k}\right\|$.

By Theorem 11 (or Theorem 12) and Theorem 2, $\mathcal{G}$ in Theorem 19 is the unique dual of $\Psi$ such that $\operatorname{dep}(\mathcal{G})=\operatorname{dep}(\mathcal{F})$.

\subsection{Frame representation of operators and the solu- tion of operator equations}

The representation of operators using bases and frames and its application to the solution of operator equations were considered in, e.g., [1, 3, 4, 6, 16, 17, 30. Here we consider them using the present general theory of dual frames.

Let $\mathcal{R}$ be a vector space over $\mathbb{F}, \mathcal{W}$ be a finite-dimensional subspace of $\mathcal{R}$. Let $\mathcal{F}=\left\{f_{i}\right\}_{i \in I} \subseteq \mathcal{V}, \Phi=\left\{\phi_{i}\right\}_{i \in I} \subseteq \mathcal{S}^{\prime}, \mathcal{G}=\left\{g_{i}\right\}_{i \in J} \subseteq \mathcal{W}$, and $\Psi=\left\{\psi_{i}\right\}_{i \in J} \subseteq \mathcal{R}^{\prime}$.

Let $\mathcal{T}_{\mathcal{F}, \Psi} \in L\left(L(\mathcal{V}, \mathcal{W}), L\left(\mathbb{F}^{I}, \mathbb{F}^{J}\right)\right)$ be defined by

$$
\mathcal{T}_{\mathcal{F}, \Psi}(L)=T_{\Psi}^{*} L T_{\mathcal{F}} \quad \text { for each } L \in L(\mathcal{V}, \mathcal{W}),
$$

and let $\mathcal{T}^{\mathcal{G}, \Phi} \in L\left(L\left(\mathbb{F}^{I}, \mathbb{F}^{J}\right), L(\mathcal{V}, \mathcal{W})\right)$ be defined by

$$
\mathcal{T}^{\mathcal{G}, \Phi}(A)=T_{\mathcal{G}} A\left(T_{\Phi}^{*}\right)_{\mid \mathcal{V}} \quad \text { for each } A \in L\left(\mathbb{F}^{I}, \mathbb{F}^{J}\right)
$$

Given $L \in L(\mathcal{V}, \mathcal{W})$, we say that $\mathcal{T}_{\mathcal{F}, \Psi}(L)$ is the linear transformation in $L\left(\mathbb{F}^{I}, \mathbb{F}^{J}\right)$ representing $L$ with respect to $\mathcal{F}$ and $\Psi$. The matrix of $\mathcal{T}_{\mathcal{F}, \Psi}(L)$ with respect to the standard bases of $\mathbb{F}^{I}$ and $\mathbb{F}^{J}$ is $\left(\psi_{j} L f_{i}\right)_{j, i}$.

If $A \in L\left(\mathbb{F}^{I}, \mathbb{F}^{J}\right)$, we say that $\mathcal{T}^{\mathcal{G}, \Phi}(A)$ is the linear transformation in $L(\mathcal{V}, \mathcal{W})$ representing $A$ with respect to $\mathcal{G}$ and $\Phi$.

We have the following result (see also [3], where $\mathcal{V}=\mathcal{S}$ and $\mathcal{W}=\mathcal{R}$ are Hilbert spaces): 
Theorem 20 Assume that $\mathcal{F}$ and $\Phi$ are dual frames in $\mathcal{V}$, while $\mathcal{G}$ and $\Psi$ are dual frames in $\mathcal{W}$. Then:

1. $\mathcal{T}^{\mathcal{G}, \Phi} \mathcal{T}_{\mathcal{F}, \Psi}=I_{L(\mathcal{V}, \mathcal{W})}$ and $L f=\sum_{j \in J} \sum_{i \in I} \phi_{i}(f) \psi_{j}\left(L f_{i}\right) g_{j}$ for each $L \in$ $L(\mathcal{V}, \mathcal{W})$ and $f \in \mathcal{V}$.

2. $\mathcal{T}^{\mathcal{G}, \Phi}$ is surjective and $\mathcal{T}_{\mathcal{F}, \Psi}$ is injective.

3. $\mathcal{T}^{\mathcal{G}, \Psi} I_{\mathbb{F}^{J}}=I_{\mathcal{W}}$.

4. Let $L \in L(\mathcal{V}, \mathcal{W}), f \in \mathcal{V}$ and $g \in \mathcal{W}$. Then $L f=g$ if and only if $\mathcal{T}_{\mathcal{F}, \Psi}(L) T_{\Phi}^{*} f=T_{\Psi}^{*} g$.

5. $\mathcal{T}_{\mathcal{F}, \Psi}(L)\left(\operatorname{im}\left(\left(T_{\Phi}^{*}\right)_{\mid \mathcal{V}}\right)\right) \subseteq \operatorname{im}\left(\left(T_{\Psi}^{*}\right)_{\mid \mathcal{W}}\right)$. If $L$ is surjective, then

$$
\mathcal{T}_{\mathcal{F}, \Psi}(L)\left(\operatorname{im}\left(\left(T_{\Phi}^{*}\right)_{\mid \mathcal{V}}\right)\right)=\operatorname{im}\left(\left(T_{\Psi}^{*}\right)_{\mid \mathcal{W}}\right) .
$$

If $L$ is injective, then $\mathcal{T}_{\mathcal{F}, \Psi}(L)_{\mid \operatorname{im}\left(\left(T_{\Phi}^{*}\right)_{\mid \mathcal{V}}\right)}$ is injective.

Given $L \in L(\mathcal{S}, \mathcal{R})$ and $g \in \mathcal{R}$, consider the operator equation

$$
L f=g .
$$

A solution of (3) can be obtained using dual frames and the Petrov-Galerkin method in the finite dimensional subspaces $\mathcal{V} \subseteq \mathcal{S}$ and $\mathcal{W} \subseteq \mathcal{R}$ such that $L(\mathcal{V}) \subseteq \mathcal{W}$. For this, we solve the finite matrix equation

$$
\mathcal{T}_{\mathcal{F}, \Psi}\left(L_{\mid \mathcal{V}}\right) c=T_{\Psi}^{*} \widetilde{g}
$$

where

- $\mathcal{F}$ and $\Phi$ are dual frames in $\mathcal{V}$ such that $\mathcal{S}=\mathcal{V} \oplus \operatorname{ker}\left(T_{\Phi}^{*}\right)$,

- $\mathcal{G}$ and $\Psi$ are dual frames in $\mathcal{W}$ such that $\mathcal{R}=\mathcal{W} \oplus \operatorname{ker}\left(T_{\Psi}^{*}\right)$,

- $\widetilde{g}=P_{\mathcal{W}, \operatorname{ker}\left(T_{\Psi}^{*}\right)} g$.

Recall that by Theorem 3, $T_{\mathcal{F}} T_{\Phi}^{*}=P_{\mathcal{V}, \operatorname{ker}\left(T_{\Phi}^{*}\right)}$ and $T_{\mathcal{G}} T_{\Psi}^{*}=P_{\mathcal{W}, \operatorname{ker}\left(T_{\Psi}^{*}\right)}$. Also, by Theorem 2, $\left.T_{\Phi}^{*} T_{\mathcal{F}}=P_{\operatorname{im}\left(T_{\Phi}^{*}\right.}^{*}\right), \operatorname{dep}(\mathcal{F})$ and $T_{\Psi}^{*} T_{\mathcal{F}}=P_{\operatorname{im}\left(T_{\Psi}^{*} \mathcal{W}^{*}\right), \operatorname{dep}(\mathcal{G})}$. Hence,

$$
T_{\Psi}^{*} \widetilde{g}=P_{\operatorname{im}\left(T_{\Psi \mid \mathcal{W}}^{*}\right) \operatorname{dep}(\mathcal{G})} T_{\Psi}^{*} g
$$

Let $c \in \mathbb{F}^{I}$ be a (possibly approximate) solution of (4) and

$$
\widetilde{c}=T_{\Phi}^{*} T_{\mathcal{F}} c=P_{\operatorname{im}\left(T_{\Phi_{\mid \mathcal{V}}}^{*}\right) \operatorname{dep}(\mathcal{F})} c .
$$

Since $\operatorname{dep}(\mathcal{F}) \subseteq \operatorname{ker}\left(\mathcal{T}_{\mathcal{F}, \Psi}\left(L_{\mid \mathcal{V}}\right)\right)$, we have

$$
\mathcal{T}_{\mathcal{F}, \Psi}\left(L_{\mid \mathcal{V}}\right) \widetilde{c}=\mathcal{T}_{\mathcal{F}, \Psi}\left(L_{\mid \mathcal{V}}\right) c
$$


We take $\tilde{f}=T_{\mathcal{F}} \widetilde{c}=T_{\mathcal{F}} c$ as a solution of (3). Assume now that $c \in \mathbb{F}^{I}$ is a true solution of (4), i.e., $\mathcal{T}_{\mathcal{F}, \Psi}\left(L_{\mid \mathcal{V}}\right) c=T_{\Psi}^{*} \widetilde{g}$. By Theorem 20, $L \widetilde{f}=\widetilde{g}$.

We can work with different types of operator equations such as differential equations or integral equations. Depending on the operator equation, we can use suitable strategies to construct the subspaces $\mathcal{V}$ and $\mathcal{W}$, and the dual frames $\mathcal{F}$ and $\Phi, \mathcal{G}$ and $\Psi$. For example, if $\mathcal{W}$ is such that $g \in \mathcal{W}$ we obtain $\widetilde{g}=g$.

\section{Acknowledgements}

The author thanks the reviewer, whose suggestions served to improve the paper. This research has been supported by Grants PIP 112-201501-00589CO (CONICET) and PROIPRO 03-1420 (UNSL).

\section{References}

[1] Z. Ambroziński and K. Rudol, Matrices defined by frames, Opuscula Math. 29 (2009), no. 4, pp. 365-375. http://dx.doi.org/10.7494/OpMath.2009.29.4.365

[2] R. Balan, Equivalence relations and distances between Hilbert frames, Proc. Am. Math. Soc. 127 (1999), no. 8, pp. 2353-2366. https://doi.org/10.1090/S0002-9939-99-04826-1

[3] P. Balazs, Matrix-representation of operators using frames, Sampl. Theory Signal Image Process. 7 (2008), no. 1, pp. 39-54. https://doi.org/10.1007/BF03549484

[4] P. Balazs and K. Gröchenig, A guide to localized frames and applications to Galerkin-like representations of operators, frames and other bases in abstract and function spaces, In: Pesenson, I., Mhaskar, H., Mayeli, A., Le Gia, Q. T., Zhou, D.-X., eds. Applied and Numerical Harmonic Analysis, Vol. 1, 2017, Basel: Birkhäuser, pp. 47-79. https://doi.org/10.1007/978-3-319-55550-8_4

[5] P. Balazs and H. Harbrecht, Frames for the Solution of Operator Equations in Hilbert Spaces with Fixed Dual Pairing, Numer. Funct. Anal. Optim. 40 (2019), no. 1, pp. 65-84. https://doi.org/10.1080/01630563.2018.1495232

[6] P. Balazs and G. Rieckh, Oversampling operators: Frame representation of operators, Analele Universitatii "Eftimie Murgu" 2 (2011), pp. 107-114. 
[7] B. G. Bodmann, M. Le, L. Reza, M. Tobin and M. Tomforde, Frame theory for binary vector spaces, Involve 2 (2009), pp. 589-602. https://doi.org/10.2140/involve.2009.2.589

[8] B. G. Bodmann, B. Camp and D. Mahoney, Binary frames, graphs and erasures, Involve 7 (2014), pp. 151-169. https://doi.org/10.2140/involve.2014.7.151

[9] P. G. Casazza, The art of frame theory, Taiwanese J. Math. 4 (2000), no. 2, pp. 129-202.

[10] P. G. Casazza and O. Christensen, Perturbation of operators and applications to frame theory, J. Fourier Anal. Appl. 3 (1997), no. 5, pp. $543-557$.

[11] P. G. Casazza and G. Kutyniok (eds.), Finite Frames. Theory and Applications, Birkhäuser, Boston, 2012.

[12] D.Y. Chen, L. Li and B.T. Zheng, Perturbations of frames, Acta Math. Sin. Engl. Ser. 30 (2014), no. 7, pp. 1089-1108.

[13] T.-Y. Chien, V. Flynn and S. Waldron, Tight frames for cyclotomic fields and other rational vector spaces, Linear Algebra Appl. 476 (2015), pp. 98-123. https://doi.org/10.1016/j.laa.2015.02.021

[14] O. Christensen, An Introduction to Frames and Riesz Bases, Second ed., Birkhäuser, Boston, 2016.

[15] O. Christensen and Y.C. Eldar, Oblique dual frames and shiftinvariant spaces, Appl. Comput. Harmon. Anal. 17 (2004), pp. 48-68. https://doi.org/10.1016/j.acha.2003.12.003

[16] S. Dahlke, M. Fornasier and T. Raasch, Adaptive frame methods for elliptic operator equations, Adv. Comput. Math. 27 (2007), no. 1, pp. 27-63. https://doi.org/10.1007/s10444-005-7501-6

[17] W. Dahmen and R. Schneider, Composite wavelet basis for operator equations, Math. Comp. 68 (1999), no. 228, pp. 1533-1567. https://doi.org/10.1090/S0025-5718-99-01092-3

[18] I. Daubechies, A. Grossman and Y. Meyer, Painless nonorthogonal expansions, J. Math. Phys. 27 (1985), pp. 1271-1283.

[19] I. Daubechies, Ten Lectures on Wavelets, SIAM, Philadelphia, PA, 1992. 
[20] R. J. Duffin and A. C. Schaeffer, A class of nonharmonic Fourier series, Trans. Amer. Math. Soc. 72 (1952), pp. 341-366. https://doi.org/10.1090/S0002-9947-1952-0047179-6

[21] Y.C. Eldar, Sampling with arbitrary sampling and reconstruction spaces and oblique dual frame vectors, J. Fourier Anal. Appl. 9 (2003), no. 1, pp. 77-96. https://doi.org/10.1007/s00041-003-0004-2

[22] K. Flornes, A. Grossmann, M. Holschneider and B. Torrésani, Wavelets on discrete fields, Appl. Comput. Harmon. Anal. 1 (1994), pp. 137-146. https://doi.org/10.1006/acha.1994.1001

[23] A. Ghaani Farashahi, Wave packet transform over finite fields, Electron. J. Linear Algebra 30 (2015), pp. 507-529. https://doi.org/10.13001/1081-3810.2903

[24] A. Ghaani Farashahi, Wave packet transforms over finite cyclic groups, Linear Algebra Appl. 489 (2016), pp. 75-92. https://doi.org/10.1016/j.laa.2015.10.001

[25] G. R. W. Greaves, J. W. Iverson, J. Jasper and D. G. Mixon, Frames over finite fields: basic theory and equiangular lines in unitary geometry, arXiv:2012.12977v1 (2020).

[26] G. R. W. Greaves, J. W. Iverson, J. Jasper and D. G. Mixon, Frames over finite fields: equiangular lines in orthogonal geometry, arXiv:2012.13642v1 (2020).

[27] R. Hotovy, D. R. Larson and S. Scholze, Binary frames, Houston J. Math. 41 (2015), no. 3, pp. 875-899.

[28] J. Kovačević and A. Chebira, An Introduction to Frames, Found. Trends Signal Process. 2 (2008), pp. 1-94.

[29] R. P. Mendez, B. G. Bodmann, Z. J. Baker, M. G. Bullok and J. E. McLaney, Binary Parseval frames from group orbits, Linear Algebra Appl. 556 (2018), pp. 265-300. https://doi.org/10.1016/j.laa.2018.07.016

[30] P. M. Morillas, Construction of orthonormal wavelet-like bases, J. Math. Phys. 51 (2010), 083510. https://doi.org/10.1063/1.3462714

[31] K. Okoudjou (ed.), Finite Frame Theory. A Complete Introduction to Overcompletness, Proc. Sympos. Appl. Math., AMS, Vol. 23, 2016.

[32] A. M. Robert, A Course in p-adic Analysis, Springer, New York, 2000. 
[33] S. Roman, Advanced Linear Algebra, Third ed., Springer, New York, 2008.

[34] R. Stevenson, Adaptive solution of operator equations using wavelet frames, SIAM J. Numer. Anal. 41 (2003), no. 3, pp. 1074-1100. https://doi.org/10.1137/S0036142902407988

[35] S. Waldron, An Introduction to Finite Tight Frames, Birkhäuser, Boston, 2018.

[36] S. Waldron, Frames for vector spaces and affine spaces, Linear Algebra Appl. 435 (2011), pp. 77-94. https://doi.org/10.1016/j.laa.2011.01.027

Patricia Mariela Morillas

Instituto de Matemática Aplicada San Luis, UNSL-CONICET

Ejército de los Andes 950, 5700 San Luis, Argentina.

morillas.unsl@gmail.com

Please, cite to this paper as published in

Armen. J. Math., V. 13, N. 2(2021), pp. 136

https://doi.org/10.52737/18291163-2021.13.2-1 